\title{
Quintessence and effective RN de Sitter brane geometries
}

\author{
K. Priyabrat Pandey ${ }^{\mathrm{a}}$, Abhishek K. Singh ${ }^{\mathrm{b}}$, Sunita Singh ${ }^{\mathrm{c}}$, Richa Kapoor $^{\mathrm{d}}$, Supriya Kar \\ Department of Physics and Astrophysics, University of Delhi, New Delhi 110 007, India
}

Received: 16 May 2014 / Accepted: 6 November 2014 / Published online: 21 November 2014

(C) The Author(s) 2014. This article is published with open access at Springerlink.com

\begin{abstract}
We revisit the effective space-time torsion curvature in a second order formalism, underlying the non-linear $U$ (1) gauge dynamics, of a two form on a $D_{4}$-brane. The formalism incorporates a significant global NS two form into the theory via its perturbative coupling to a dynamical two form. In particular we explore the non-linear gauge dynamics on a $D_{4}$-brane in the presence of a non-trivial background metric. An effective de Sitter universe is shown to emerge on a vacuum created pair of $(D \bar{D})_{3}$-branes by a local two form at the past horizon with the Big Bang. We obtain a number of $4 D$ de Sitter quantum black holes with and without a propagating torsion. In a low energy limit the non-perturbative correction may be seen to be insignificant. The quantum black hole undergoes an expansion in the limit and identifies with the Einstein vacuum. Interestingly our analysis reveals a plausible quintessence (axion) on an anti- $D_{3}$-brane which may source the dark energy in a $D_{3}$-brane universe. Arguably a brane universe moves away from its anti-brane due to the conjectured repulsive gravity underlying the quintessence. It leads to a growth in the extra fifth dimension between a brane and an anti-brane, which may provide a clue to an accelerating universe as observed in cosmology.
\end{abstract}

\section{Introduction}

Dirichlet $p$-branes ( $D_{p}$-branes) have been established as the non-perturbative objects due to their Ramond-Ramond (RR) charges in type IIA or IIB superstring theory in ten dimensions [1]. The non-linear brane world-volume gauge dynamics is approximated by the Dirac-Born-Infeld (DBI) action. Since closed strings are tangential to a $D_{p}$-brane, Einstein

\footnotetext{
a e-mail: kppandey@physics.du.ac.in

b e-mail: abhishek151983@gmail.com

c e-mail: sunita@physics.du.ac.in

d e-mail: richa.phy@du.ac.in

e e-mail: skkar@physics.du.ac.in
}

gravity is believed to decouple from a $D_{p}$-brane world volume [2]. Nevertheless a non-linear $U(1)$ gauge field is well known to describe an effective open string metric on a $D_{p^{-}}$ brane and leads to a number of near horizon geometries [3-15]. In fact a constant Neveu-Schwarz (NS) two form background in an open string theory, in combination with an electromagnetic field, forms a gauge invariant field strength which governs the non-linear $U(1)$ charge on a $D_{p}$-brane. The effective geometries on a $D_{p}$-brane presumably lead us to investigate the underlying effective (space-time) curvature theory in a second order formalism unparalleled to Einstein gravity.

In the context it has been conjectured that a dynamical NS two form in an open string theory in principle may lead to a generalized non-commutative geometry on a $D_{p}$-brane. However, the perspective of a dynamical two form on a $D_{p^{-}}$ brane has not been addressed due to some of the technical difficulties. In the recent past we have explored a plausible effective gravity scenario, underlying a two form dynamics, on a $D_{4}$-brane [16-22]. Primarily the Poincaré duality has played a significant role to identify a gauge theoretic torsion $H_{3}$ underlying the $U(1)$ gauge symmetry on a $D_{4}$-brane [23, $24]$. Generically a dynamical higher form on a higher $(p>3)$ dimensional $D_{p}$-brane may be seen to redefine an open string effective metric. However, in our analysis we have focused on a dynamical two form in the $U$ (1) gauge theory on a $D_{4}$-brane to construct a geometric torsion $\mathcal{H}_{3}$ in a five dimensional world volume underlying a non-perturbative curvature. In fact, a perturbative global NS two form coupling, to a local two form via its gauge curvature, has been shown to modify the covariant derivative appropriately in an underlying gauge theory. Alternately the world volume of a $D_{4}$-brane may be approximated by the dynamics of a geometric torsion in an effective space-time curvature conceptualized in a second order formalism. The $U$ (1) gauge invariance(s), underlying an effective curvature in a non-perturbative formalism and a non-linear gauge curvature in a perturbative theory, are checked to be restored independently. The two form $U(1)$ 
gauge invariance of the effective curvature scalar ensures a metric fluctuation and hence describes space-time in the formalism.

On the other hand a torsion is well known to play a significant role in the folk lore of string theories $[25,26]$. The torsion geometries leading to various quantum black holes in five dimensions have been obtained [16]. It is argued that the global modes of a NS two form in the string background may play an important role to describe a constant vacuum energy density in an effective curvature formalism. Thus a propagating two form together with the NS non-propagating modes are sensibly addressed in an effective curvature scalar $\tilde{\mathcal{K}}$ underlying a geometric torsion. It was shown that in a gauge choice for a non-propagating torsion, the effective space-time on a $S^{1}$ may be identified with the Riemannian geometry [17]. In fact the emergent brane geometries are analyzed for a torsion potential for its dependence on radial coordinate to confirm their stringy feature. In a low energy limit the effective torsion potential may be ignored. The quantum brane geometries in the limit presumably identify with some of the Einstein vacua.

Interestingly a space-time (quantum) fluctuation on a $D_{4}$ brane in an effective curvature theory may alternately be viewed through a pair creation of a $D_{3}$-brane and an anti$D_{3}$-brane ( $\bar{D}_{3}$-brane) at a vacuum in a non-linear $U(1)$ gauge theory. For instance, we consider a dynamical two form in a $U(1)$ gauge theory on a $D_{4}$-brane in the presence of a nontrivial background metric sourced by the global modes of NS two form in the open string bulk. The background metric for a $D_{4}$-brane gauge dynamics presumably describes a hidden $\bar{D}_{4}$-brane in the formalism. A priori, the $D_{4}$-brane vacuum may be seen to be governed by an effective black hole essentially sourced by the global modes of a NS two form [3-15]. Then the two form in the gauge theory may be seen to vacuum create a pair of $(D \bar{D})_{3}$-branes at the past horizon of a background pure de Sitter. The pair creation by a two form in the gauge theory on a $D_{4}$-brane is analogous to a charged particle and an anti-particle pair production by a photon at the event horizon of a background black hole [27]. The mechanism incorporates the quantum gauge theoretic effects into a classical black hole and enables one to address a semi-classical black hole and Hawking radiations. In our effective curvature formalism, the dynamical spacetime has been argued to be created at the Big Bang singularity which turns out to describe a past horizon [16]. In other words, a dynamical two form in a $U(1)$ gauge theoretic vacuum on a $D_{4}$-brane creates a pair of $(D \bar{D})_{3}$-branes and they move away from the black hole event horizon with opposite momentum. The emergent space-time on a pair of brane/antibrane is essentially sourced by an intrinsic non-linearity in a dynamical two form. An axion, Poincaré dual to a torsion, on a $\bar{D}_{3}$-brane may be seen to describe a quintessence underlying a varying vacuum energy density observed by CMB in cosmology. For instance, see references [28-30] for some of the recent developments with the idea of quintessence in literature. Qualitative analysis leading to a quintessence in the formalism may be seen to be sourced by the hidden fifth dimension between a pair of vacuum created $(D \bar{D})_{3}$-branes.

Furthermore the coupling of a global mode of NS two form to a gauge theoretic torsion ensures an emergent dynamical space-time via a metric fluctuation. Thus an effective geometry on a $D_{4}$-brane may alternately be viewed via a vacuum created pair of $(D \bar{D})_{3}$-brane universe separated by an extra fifth dimension transverse to their four dimensional world volumes. Generically a pair of lower dimensional brane/antibrane space-time, from a higher dimensional $D$-brane vacuum, breaks supersymmetry and leads to a non-BPS D-brane configuration in an effective curvature formalism. Nevertheless from the perspective of a perturbative gauge theory, it may be revealing to analyze an appropriate Higgs mechanism underlying a spontaneous symmetry breaking. The broken symmetry phase associated with the massive modes may describe a vacuum created pair of $(D \bar{D})_{3}$-branes.

In the paper we obtain effective de Sitter brane geometries by geometric engineering of the ansatz for the global modes of a two form and a local two form. In particular we consider two different plausible $4 D$ scenario underlying a torsion free universe and a new universe in the presence of a dynamical geometric torsion. The source potential in the de Sitter-like black holes on a vacuum created $D_{3}$-brane and anti-brane hint at a hidden fifth dimension in the formalism.

On the one hand a torsion free universe is a special case underlying a gauge choice in the effective torsion curvature formalism. In the case we obtain a Schwarzschild-de Sitter and a topological de Sitter-like black holes in $4 D$ with a hint for an extra dimension. The effective de Sitter vacua are further analyzed for their angular velocities, event horizon and curvature singularities if any. We redefine the background, global NS two form, charge by absorbing the torsion charge and obtain a typical $4 D$ Schwarzschild-like black hole in the effective curvature formalism. For instance, we refer to references [31-42] for an extensive review on various recent developments in de Sitter geometries and inflationary string cosmology.

On the other hand a non-trivial torsion in the formalism is shown to modify the background de Sitter vacuum. The causal geometric patches in the brane geometry may appropriately be rearranged to yield an effective ReissnerNordstorm- (RN-) like de Sitter in the presence of a perturbative correction underlying a gauge theoretic torsion charge coupled to a flat space-time. A priori the RN-like de Sitter case shall be seen to be sourced by three independent potentials underlying three non-linear charges in the formalism. They are: (1) the global modes of a NS two form, (2) an axion (Poincaré dual to a gauge theoretic $\mathrm{H}_{3}$ ) and (3) a geometric torsion. It is needless to mention that a non-linear 
charge sourced by a geometric torsion ensures the coupling of a global NS two form with a local two form. The charge underlying a geometric torsion associates a stringy feature with the effective RN-like de Sitter on a vacuum created pair of $(D \bar{D})_{3}$-branes. The torsion charge is renormalized at the past horizon to describe a topological de Sitter like black hole on a brane world.

We plan the paper as follows. We begin with a moderate introduction in Sect. 1. It is followed by an out-line on a geometric torsion underlying a second order curvature formalism in Sect. 2. An effective geometry scenario on a vacuum created pair of $(D \bar{D})_{3}$-branes at the Big Bang by a two form, in the presence of a background metric, in a $U(1)$ gauge theory on a $D_{4}$-brane is presented in Sect. 3 . An axion on $\bar{D}_{3}$-brane, possessing its origin in a torsion, is qualitatively analyzed for its dual role leading to: (1) a quintessence vacuum energy density in Sect. 3.1 and (2) a D-instanton (non-perturbative) correction to the perturbative vacuum in Sect. 3.2. We work out the effective de Sitter torsion free geometries on a brane in Sect. 4 which are shown to describe a Schwarzschildde Sitter and a topological de Sitter in $4 D$ with a hidden fifth dimension. In Sect. 5 we work out new quantum de Sitter geometries underlying a propagating torsion. A stringy $\mathrm{RN}$ de Sitter black hole and a renormalization of a torsion charge leading to topological de Sitter are obtained under the new geometries. We conclude in Sect. 6 by summarizing the results with a plausible remark on a quintessence (axion) in the formalism.

\section{Preliminaries}

\subsection{Geometric torsion $\mathcal{H}_{3}$ in 5D}

A $D_{4}$-brane is a dynamical object in ten dimensional type IIA superstring theory. Its five dimensional world-volume dynamics may be approximated by a Dirac-Born-Infeld (DBI) action which describes a non-linear one form $\mathcal{A}_{\mu}$ in a $U$ (1) gauge theory. For a constant background metric $g_{\mu \nu}$ the $\mathcal{A}_{\mu}$ dynamics, leading to a non-linear field strength,

$$
\bar{F}_{\mu \nu}=\left(2 \pi \alpha^{\prime}\right) F_{\mu \nu}=\left(\bar{F}_{\mu \nu}^{\text {linear }}+B_{\mu \nu}^{\text {global }}\right)
$$

is given by

$$
S=-\frac{1}{4 C_{1}^{2}} \int d^{5} x \sqrt{-\operatorname{det}(g+\bar{F})_{\mu \nu}}
$$

where $C_{1}^{2}=\left(4 \pi^{2} g_{s}\right) \alpha^{\prime 5 / 2}$. Alternately the Poincaré dual to the field strength on a $D_{4}$-brane underlie a dynamical two form $B_{\mu \nu}$ and is well known to incorporate a gauge theoretic torsion $H_{3}=d B_{2}$. The two form gauge dynamics is given by

$$
\begin{gathered}
S=-\frac{1}{12 C_{2}^{2}} \int d^{5} x \sqrt{-g} H_{\mu \nu \lambda} H^{\mu \nu \lambda}, \\
\text { where } C_{2}^{2}=\left(8 \pi^{3} g_{s}\right) \alpha^{\prime 3 / 2} .
\end{gathered}
$$

Interestingly the significance of the global modes of the NS two form in addition to the local modes of a two form have been addressed in an effective curvature formalism on a $D_{4}$ brane $[16,17]$. A priori the gauge theoretic torsion $H_{3}$ has been identified with a connection in the formalism leading to a modified covariant derivative $\mathcal{D}_{\mu}$ on a brane. The gauge connections are appropriately coupled to a two form and have been shown to define a geometric torsion $\mathcal{H}_{3}$ which takes the usual form:

$\mathcal{H}_{\mu \nu \lambda}=\mathcal{D}_{\mu} B_{\nu \lambda}+$ cyclic in $(\mu, \nu, \lambda)$,

where $\mathcal{D}_{\lambda} B_{\mu \nu}=\nabla_{\lambda} B_{\mu \nu}+\frac{1}{2} H_{\lambda \mu}^{\rho} B_{\rho \nu}-\frac{1}{2} H_{\lambda \nu}^{\rho} B_{\rho \mu}$.

An iterative incorporation of $B_{2}$-corrections to all orders in the covariant derivative leads to an exact derivative in a perturbative gauge theory. It may be seen to define a nonperturbative covariant derivative in a second order formalism underlying a geometric realization. Then the geometric torsion may be given by

$$
\begin{aligned}
\mathcal{H}_{\mu \nu \lambda}= & \nabla_{\lambda} B_{\mu \nu} !+\frac{1}{2} \mathcal{H}_{\lambda \mu}{ }^{\rho} B_{\rho \nu}-\frac{1}{2} \mathcal{H}_{\lambda \nu}{ }^{\rho} B_{\rho \mu} \\
& +\operatorname{cyclic} \text { in }(\mu, v, \lambda), \\
= & H_{\mu \nu \lambda}+3 \mathcal{H}_{[\mu \nu}{ }^{\alpha} B^{\beta}{ }_{\lambda]} g_{\alpha \beta} \\
= & H_{\mu \nu \lambda}+\left(H_{\mu \nu \alpha} B^{\alpha}{ }_{\lambda}+\text { cyclic in } \mu, \nu, \lambda\right) \\
& +H_{\mu \nu \beta} B^{\beta}{ }_{\alpha} B^{\alpha}{ }_{\lambda}+\cdots .
\end{aligned}
$$

The $U(1)$ gauge invariance of a geometric torsion under a two form transformation shall explicitly be realized in an effective space-time curvature formalism. Nevertheless in the modified $U(1)$ gauge theory a global two form acts as a perturbation parameter and is distinctly placed rather than the local two form. Thus the gauge invariance $\delta_{\mathrm{nz}} \mathcal{H}_{3}=0$ may be retained by the non-zero modes of the two form as $\delta_{\mathrm{nz}} H_{3}=0$ and $\delta_{\mathrm{nz}} B^{\text {global }}=0$.

\subsection{Space-time curvature on a $\mathbf{D}_{\mathbf{4}}$-brane}

Interestingly, the $B_{2}$-field dynamics in a first order formalism may be viewed as a torsion dynamics on an effective $D_{4}$ brane in a second order formalism [16], leading to a fourth order generalized tensor:

$$
\begin{aligned}
4 \tilde{\mathcal{K}}_{\mu \nu \lambda}{ }^{\rho}= & 2 \partial_{\mu} \mathcal{H}_{\nu \lambda}{ }^{\rho}-2 \partial_{\nu} \mathcal{H}_{\mu \lambda}{ }^{\rho}+\mathcal{H}_{\mu \lambda}{ }^{\sigma} \mathcal{H}_{\nu \sigma}{ }^{\rho} \\
& -\mathcal{H}_{\nu \lambda}{ }^{\sigma} \mathcal{H}_{\mu \sigma}{ }^{\rho} .
\end{aligned}
$$

The generalized tensor is antisymmetric under the exchange of indices within a pair and is not symmetric under the exchange of its first pair of indices and the second. Hence, it differs from the Riemannian tensor $R_{\mu \nu \lambda \rho}$. However, for 
a non-propagating torsion $\tilde{\mathcal{K}}_{\mu \nu \lambda \rho} \rightarrow R_{\mu \nu \lambda \rho}$. The second order curvature tensor and the irreducible curvature scalar are worked out:

$$
\begin{aligned}
4 \tilde{\mathcal{K}}_{\mu \nu} & =-\left(2 \partial_{\lambda} \mathcal{H}_{\mu \nu}{ }^{\lambda}+\mathcal{H}_{\mu \rho}{ }^{\lambda} \mathcal{H}_{\lambda \nu}{ }^{\rho}\right) \\
\text { and } \quad \tilde{\mathcal{K}} & =-\frac{1}{4} \mathcal{H}_{\mu \nu \lambda} \mathcal{H}^{\mu \nu \lambda} .
\end{aligned}
$$

As a result, the effective curvature constructed in a nonperturbative framework may be viewed as a generalized curvature tensor. In a second order formalism the effective $\mathcal{H}_{3}$ dynamics on a $D_{4}$-brane may be approximated by

$S_{\mathrm{D}_{4}}^{\text {eff }}=\frac{1}{3 C_{2}^{2}} \int d^{5} x \sqrt{-\operatorname{det} \tilde{G}_{\mu \nu}} \tilde{\mathcal{K}}$.

Generically the open string metric may arguably be reexpressed in terms of the Poincaré dual field strength. It takes the form

$\tilde{G}_{\mu \nu}=\left(\tilde{G}_{\mu \nu}^{z}+C \overline{\mathcal{H}}_{\mu \lambda \rho} \mathcal{H}^{\lambda \rho}{ }_{\nu}\right)$,

where $\tilde{G}_{\mu \nu}^{z}=\left(g_{\mu \nu}-B_{\mu \lambda} B_{\nu}^{\lambda}\right)$ and $B_{\mu \nu}$ signify the global or zero modes of the two form, which are indeed coupled to an electromagnetic field $F_{\mu \nu}^{\text {linear }}$. Under a two form gauge transformation $\delta B_{\mu \nu}=\left(\partial_{\mu} \epsilon_{\nu}-\partial_{\nu} \epsilon_{\mu}\right)$ the $U$ (1) gauge invariance underlying the curvature scalar $\tilde{\mathcal{K}}$ is worked out explicitly:

$$
\begin{aligned}
\delta \tilde{\mathcal{K}}= & \frac{3}{2} \delta\left(\mathcal{D}_{[\mu} B_{\nu \lambda]}\right) \mathcal{H}^{\mu \lambda \nu} \\
= & \frac{1}{2} \delta\left(H_{\mu \nu \lambda}+H_{\mu \nu}{ }^{\rho} B_{\rho \lambda}+H_{\nu \lambda}{ }^{\rho} B_{\rho \mu}\right. \\
& \left.+H_{\lambda \mu}{ }^{\rho} B_{\rho \nu}\right) \mathcal{H}^{\mu \lambda \nu} \\
= & \frac{3}{2} H_{\mu \nu}{ }^{\rho} \delta B_{\rho \lambda} \mathcal{H}^{\mu \lambda \nu} \\
= & \frac{3}{2}\left(H_{\mu \nu}{ }^{\lambda} \mathcal{H}^{\mu \nu \rho}-H_{\mu \nu}{ }^{\rho} \mathcal{H}^{\mu \nu \lambda}\right) \partial_{\rho} \epsilon_{\lambda} .
\end{aligned}
$$

The $U(1)$ gauge invariance, i.e. $\delta \tilde{\mathcal{K}}=0$, may be achieved by the notion of a metric (fluctuation) sourced by the local degrees in a geometric torsion. A priori the gauge invariance of the curvature scalar $\tilde{\mathcal{K}}$ seems to incorporate a condition on the two form:

$C\left(2 \pi \alpha^{\prime}\right) H_{\alpha \beta}^{\mu} \mathcal{H}^{\alpha \beta v}=f_{q}^{\mu \nu}$.

Nevertheless the perturbation gauge theory ensures an infinitesimally small global (two form) mode coupling in a geometric torsion (5) and hence the condition may be ignored. Then the emerging notion of a metric underlying a propagating geometric torsion in the non-perturbative curvature formalism is given by

$f_{\mu \nu}^{q}=C \mathcal{H}_{\mu \alpha \beta} \overline{\mathcal{H}}_{\nu}^{\alpha \beta}$.

The symmetry under an interchange $\mu \leftrightarrow v$ in the r.h.s. ensures that the naive condition on a two form becomes insignificant. Interestingly the emergent metric incorporates the notion of space-time in the curvature scalar $\tilde{\mathcal{K}}$ and hence justifies the open string effective metric arguably obtained in Eq. (9) on a $D_{4}$-brane.

\section{Vacuum created pair of $(\mathrm{DD})_{3}$-brane}

It is remarkable to note that an effective space-time is vacuum created on a pair of $(D \bar{D})_{3}$-branes by a dynamical two form in a $U(1)$ gauge theory on a $D_{4}$-brane. The vacuum refers to a background black hole on a $D_{4}$-brane which is well known to be sourced by a global mode of a NS two form. Hence a geometric torsion underlying the second order curvature formalism is associated with a universe with a vacuum created pair of $(D \bar{D})_{3}$-branes. In fact, the dynamical space-time without torsion in $4 D$ has been argued to have begun at the Big Bang singularity defined at a cosmological horizon in a 5D-brane world with torsion. It is noteworthy to remark that the space-time curvature sourced by a geometric torsion in $5 D$ naturally incorporates an intrinsic angular momentum on a vacuum created pair of $(D \bar{D})_{3}$-branes. The world volumes of a brane and an anti-brane are separated by an extra fifth dimension transverse to each other. It may imply that an observer in a $D_{3}$-brane universe is inaccessible to an anti$D_{3}$-brane universe or vice versa. Nevertheless the invisible effect of an anti- $D_{3}$-brane space-time is indeed compelling on a 3-brane universe due to the hidden fifth dimension. Analysis may reveal that the brane universe geometry seems to be influenced by a potential candidate for dark energy.

\subsection{Quintessence}

The CMB observations in cosmology ensure that the vacuum energy density is not a constant. The time variation of the cosmological vacuum energy density in the early epoch leading to a de Sitter geometry has been argued in the inflationary models. In this context a quintessence is well known to describe a time varying, spatially homogeneous component of cosmic density with a negative pressure and a positive energy density. In the recent past a slowly rolling time dependent scalar field is believed to describe the quintessence presumably due to its simplest form as a tensor field which couples minimally to Einstein gravity. The acceleration of the universe is parameterized by the cosmological equation of state: $\omega=P / \rho$, where $P=-T_{i}^{i}<0$ and $\rho=T_{0}^{0}>0$, respectively, define the pressure and energy density sourced by a scalar field. In the recent years the observational cosmology primarily has focused on the measurement of $\omega$ sourced by a quintessence. It takes a bounded value: $-1<\omega<-1 / 3$.

The quintessence energy density underlying geometric torsion in the formalism may be seen to be sourced by a 
dynamical two form. For instance, a non-trivial curvature $\mathcal{K}$ may be seen to be sourced by a dynamical mass-less axion on an anti- $D_{3}$-brane. The presence of an extra fifth dimension transverse to the anti-brane effects a vacuum created $D_{3}$-brane universe which is described by a non-linear two form $\mathcal{F}_{2}$. The inaccessible scalar dynamics to an observer in an effective space-time on a $D_{3}$-brane provokes one to identify the Poincaré dual of torsion as a quintessence scalar field in the formalism. The idea of a quintessence energy density sourced by an axion field on an anti-brane is remarkable. A quintessence scalar field is believed to be a potential candidate to describe the dark energy in our universe. The fact that the dark energy permeates all space and tends to enhance the rate of expansion of universe may be understood via an extra transverse dimension between a pair of vacuum created $D_{3}$-brane and $\bar{D}_{3}$-brane in the formalism. Hubble's law may be revisited to explain the receding of a brane from an antibrane. The law states that the recessional speeds of brane and anti-brane universes are proportional to the transverse distance between them. Since a vacuum created brane universe moves away from its an anti-brane, in an opposite direction, it implies a repulsive effective gravity between them. The notion of repulsive gravity has been argued to be sourced by a quintessence scalar field in cosmology. One is tempted to believe that an axion in a hidden anti-brane is realized through a geometric torsion which in turn is responsible for the repulsive gravity. In fact, repulsive gravity underlying quintessence has been conjectured between matter and antimatter which may be seen to be in conformity with the notion of a vacuum created pair of $(D \bar{D})_{3}$-branes by a two form on a $D_{4}$-brane. Interestingly the effective de Sitter black holes, obtained in the gauge choice for a non-propagating torsion, may be seen to be described with a varying positive curvature scalar $R=12 / r^{4}$. It further strengthens the presence of the quintessence axion in the formalism. It may imply that the brane/anti-brane universe(s) at its vacuum creation, i.e. at the past horizon radius $b$, with a Big Bang, was described by a constant curvature $R=12 / b^{4}>0$. At the Big Bang the quintessence kinetic energy is ignored and hence the equation of state reduces to $\omega=-1$. The result is in agreement with the value of $\omega$ known for a constant vacuum energy density.

\subsection{D-instanton correction}

An axion in the RR sector of the type II superstring hints to a D-instanton in the formalism. Importantly a D-instanton is known to incorporate a non-perturbative correction in a perturbative string theory. In fact the universe was argued to begin at a past horizon with the Big Bang on a pair of $(D \bar{D})$ instantons at an early epoch in the de Sitter case underlying the geometric torsion formalism [16]. The hot de Sitter phase is believed to expand and the notion of a real time was incor- porated subsequently in the geometry. The expansion of the universe via a pair of branes (or anti-branes) from its origin at the Big Bang was argued to go through a series of nucleations of a pair of $D$-instantons followed by higher dimensional pairs: $D$-particle, $D$-string, $D$-membrane, and the process stops with a pair of $D_{3}$-branes in the formalism. It is due to the fact that a pair of $(D \bar{D})_{3}$-branes with a transverse fifth dimension completely fills the $D_{4}$-brane world volume.

In other words an instanton local degree on an anti- $D_{3}$ brane influences the world volume of a $D_{3}$-brane within a pair. Arguably the instantaneous effect of a $\bar{D}$-instanton on a $D_{3}$-brane is in agreement with the non-perturbative universe in $4 D$. For $\kappa^{2}=(2 \pi)^{5 / 2} g_{s} \alpha^{\prime}$, the non-perturbative curvature dynamics on $S^{1}$ takes the form

$S=\frac{1}{3 \kappa^{2}} \int d^{4} x \sqrt{-\operatorname{det} G_{\mu \nu}} \quad\left(\mathcal{K}-\frac{3}{4} \overline{\mathcal{F}}_{\mu \nu} \mathcal{F}^{\mu \nu}\right)$,

where $\quad \mathcal{F}_{\mu \nu}=F_{\mu \nu}+\mathcal{H}_{\mu \nu}^{\lambda} \mathcal{A}_{\lambda}$.

The curvature scalar $\mathcal{K}$ is essentially sourced by a dynamical two form potential in a first order formalism. Its field strength is appropriately modified to define a dynamical geometric torsion $\mathcal{H}_{3}$ in four dimensions underlying a second order space-time curvature formalism. The fact that a torsion is dual to an axion (scalar) on an effective $D_{3}$-brane ensures one degree of freedom. In addition $\mathcal{F}_{\mu \nu}$ describes a geometric one form field with two local degrees on an effective $D_{3}$-brane. A precise match among the (three) local degrees of torsion in $\tilde{\mathcal{K}}$ on $S^{1}$ with that in $\mathcal{K}$ and $\mathcal{F}_{\mu \nu}$ ensures the absence of a dynamical dilation field in the framework. The result is consistent with the fact that a two form on $S^{1}$ does not generate a dilaton field. Alternately an emergent metric tensor, essentially sourced by a dynamical two form, does not formally evolve under compactification of an underlying $U(1)$ gauge theory (8) on $S^{1}$.

In the absence of a geometric torsion, Eq. (14) ensures a gauge theoretic non-linear field strength on a $D_{3}$-brane. Intuitively a geometric torsion leading to a spinning $D_{3}$-brane presumably enforces the notion of an anti- $D_{3}$-brane spinning in an opposite direction to the brane. In a low energy limit the spin slows down significantly and may be ignored to retain the BPS configuration, i.e. a BPS brane and an antiBPS brane. In the limit the extra fifth dimension becomes large and hence a brane universe and an anti-brane universe may appear to be independent of each other. In the case the quintessence field dynamics freezes on an anti-brane and the brane universe is precisely governed by two local degrees of the gauge theoretic non-linear one form.

On the other hand the energy-momentum tensor $T_{\mu \nu}$ is computed in a gauge choice:

$\frac{3}{4} \overline{\mathcal{F}}_{\mu \nu} \mathcal{F}^{\mu \nu}=\frac{3}{\pi \alpha^{\prime}}+\mathcal{K}$.

Explicitly 
$\left(2 \pi \alpha^{\prime}\right)^{2} T_{\mu \nu}=\left(G_{\mu \nu}^{z}+\tilde{C} \overline{\mathcal{F}}_{\mu \lambda} \overline{\mathcal{F}}^{\lambda}{ }_{\nu}+C \overline{\mathcal{H}}_{\mu \lambda \rho} \mathcal{H}^{\lambda \rho}{ }_{\nu}\right)$.

The gauge choice ensures that the $T_{\mu \nu}$ sources a non-trivial emergent geometry underlying a non-linear $U(1)$ gauge theory. Interestingly the $T_{\mu \nu}$ sources an effective metric $G_{\mu \nu}$ in the framework. $T_{\mu \nu}$ with $\left(C=\frac{3}{4} \tilde{C}=\frac{3}{2}\right)$ and $\left(C=-\frac{5}{4}\right.$, $\left.\tilde{C}=-\frac{1}{2}\right)$, respectively, corresponds to a $(+)$ ve sign and a $(-)$ ve sign in $G_{\mu \nu}$. They describe two inequivalent black holes on an effective $D_{3}$-brane. To take two solutions for an emergent metric tensor is a possible choice, keeping the generality in mind. Primarily they dictate the quantum geometric corrections to a background metric. Generically the effective metric on a $D_{p}$-brane for $p \neq 4$ may be given by

$G_{\mu \nu}=\left(G_{\mu \nu}^{z} \pm \overline{\mathcal{F}}_{\mu \lambda} \overline{\mathcal{F}}^{\lambda}{ }_{\nu} \pm \overline{\mathcal{H}}_{\mu \lambda \rho} \mathcal{H}^{\lambda \rho}{ }_{\nu}\right)$.

The generalized metric incorporate the local modes of a two form in addition to the global modes of a NS two form from the open string bulk. Torsion may be seen to incorporate conserved quantities, i.e. angular momentum and/or a charge, on a brane world. Interestingly the torsion braneworld geometries become significant on the Planck energy scale and may be identified with primordial rotating and/or charged black holes and their higher dimensional generalizations.

\section{Quantum de Sitter brane in 4D without torsion}

\subsection{Two form ansatz}

A geometric torsion underlying a $U(1)$ gauge theory on a $D_{4}$-brane may alternately be viewed via a vacuum created pair of the $(D \bar{D})_{3}$-branes. Within a pair the world volumes are distinctly separated by an extra fifth dimension. In other words an irreducible scalar curvature $\tilde{\mathcal{K}}$ on $S^{1}$ generically reduces to $\mathcal{K}$ on a $D_{3}$-brane and a geometric $\mathcal{F}_{2}$ on an anti$D_{3}$-brane or vice versa. The two form ansatz in $4 D$ may be given by

$$
\begin{aligned}
B_{t \theta} & =B_{r \theta}=\frac{b}{\sqrt{2 \pi \alpha^{\prime}}}, \\
B_{\theta \phi} & =\frac{p}{\sqrt{2 \pi \alpha^{\prime}}} \sin ^{2} \theta, \\
\mathcal{A}_{t} & =\frac{Q_{e}}{r}
\end{aligned}
$$

and $\quad \mathcal{A}_{\phi}=-\frac{Q_{m}}{\sqrt{2 \pi \alpha^{\prime}}} \cos \theta$,

where $(b, p)>0$ are constants and $\left(Q_{e}, Q_{m}\right)$ denote the non-linear (electric, magnetic) charges. The gauge choice ensures a vanishing torsion $H_{3}=0=\mathcal{H}_{3}$ in the presence of the $B_{2}$-fluctuations. The two form field strength is worked out for its non-vanishing components to yield
$\mathcal{F}_{0 r}=\frac{Q_{e}}{r^{2}} \quad$ and $\quad \mathcal{F}_{\theta \phi}=\frac{Q_{m}}{2 \pi \alpha^{\prime}} \sin \theta$.

The emergent quantum geometry in the low energy limit may describe a four dimensional Einstein vacuum. The open string effective metric sourced by a constant two form may be seen to receive further geometric corrections due to the non-linear $U$ (1) charges underlying a generic $\mathcal{F}_{2}$ on a $D_{3}$-brane. Generically the emergent metric may be expressed as

$G_{\mu \nu}=\left(g_{\mu \nu} \pm B_{\mu \lambda} g^{\lambda \rho} B_{\rho \nu} \pm \overline{\mathcal{F}}_{\mu \lambda} g^{\lambda \rho} \overline{\mathcal{F}}_{\rho \nu}\right)$.

For simplicity we set $\left(2 \pi \alpha^{\prime}\right)=1$. Explicitly the line element(s) underlying the non-trivial brane geometries are given by

$$
\begin{aligned}
d s^{2}= & -\left(1-\frac{b^{2}}{r^{2}} \pm \frac{Q_{e}^{2}}{r^{4}}\right) d t^{2}+\left(1+\frac{b^{2}}{r^{2}} \pm \frac{Q_{e}^{2}}{r^{4}}\right) d r^{2} \\
& +\frac{2 b^{2}}{r^{2}} d t d r-\frac{2 b p \sin ^{2} \theta}{r^{2}}(d t+d r) d \phi \\
& +\left(1+\frac{p^{2} \sin ^{2} \theta \mp Q_{m}^{2}}{r^{4}}\right) r^{2} d \Omega^{2}
\end{aligned}
$$

and

$$
\begin{aligned}
d s^{2}= & -\left(1+\frac{b^{2}}{r^{2}} \pm \frac{Q_{e}^{2}}{r^{4}}\right) d t^{2}+\left(1-\frac{b^{2}}{r^{2}} \pm \frac{Q_{e}^{2}}{r^{4}}\right) d r^{2} \\
& -\frac{2 b^{2}}{r^{2}} d t d r+\frac{2 b p \sin ^{2} \theta}{r^{2}}(d t+d r) d \phi \\
& +\left(1-\frac{p^{2} \sin ^{2} \theta \pm Q_{m}^{2}}{r^{4}}\right) r^{2} d \Omega^{2}
\end{aligned}
$$

\subsection{Hot de Sitter brane geometries}

We perform an appropriate Weyl scaling of the emergent metric to access de Sitter vacua without any change in causal properties. It is given by

$\tilde{G}_{\mu \nu}=\lambda(r) G_{\mu \nu}$,

where $\lambda=b^{2} / r^{2}$. For an allowed range of parameters $b>r>Q_{e} / b>0$, the conformal factor $\lambda>1$. The upper bounds on $r$ and $Q_{e}$ further enforce an ultra-high energy scale in the transformed vacua. Though it implies a critical value for an electric field it does not bound a magnetic field in the quantum vacua. Thus the magnetic field can be infinitely large and may be seen to incorporate a rotation into the geometries. The stringy geometries are worked out on a vacuum created pair of a $(D \bar{D})_{3}$-brane by a two form on a $D_{4}$-brane. They are given by

$$
\begin{aligned}
d s^{2}= & -\left( \pm 1+\frac{r^{2}}{b^{2}} \pm \frac{Q_{e}^{2}}{r^{2} b^{2}}\right) d t^{2} \pm \frac{2 p}{b}(d t+d r) \sin ^{2} \theta d \phi \\
& +\left(\mp 1+\frac{r^{2}}{b^{2}} \pm \frac{Q_{e}^{2}}{r^{2} b^{2}}\right) d r^{2} \mp 2 d r d t
\end{aligned}
$$




$$
+\frac{r^{4}}{b^{2}}\left(1 \mp \frac{p^{2} \sin ^{2} \theta+Q_{m}^{2}}{r^{4}}\right) d \Omega^{2} .
$$

We consider a (-)ve sign with a two form fluctuation in the emergent metric (19). In this case the stringy vacua may be given by

$$
\begin{aligned}
d s^{2}= & -\left(-1+\frac{r^{2}}{b^{2}} \pm \frac{Q_{e}^{2}}{r^{2} b^{2}}\right) d t^{2}-\frac{2 p}{b}(d t+d r) \sin ^{2} \theta d \phi \\
& +\left(1+\frac{r^{2}}{b^{2}} \pm \frac{Q_{e}^{2}}{r^{2} b^{2}}\right) d r^{2}+2 d r d t \\
& +\left(\rho_{+}^{2} \mp N_{m}^{2}\right) d \Omega^{2} .
\end{aligned}
$$

The modified radii of $S_{2}$ are $\rho_{ \pm}=\frac{1}{b} \sqrt{r^{4} \pm p^{2} \sin ^{2} \theta}$. In a brane regime $b>r>N_{e}$ the stringy geometries describe de Sitter black holes with euclidean signature on a $D_{3}$-brane. They are given by

$$
\begin{aligned}
d s^{2}= & \left(1-\frac{r^{2}}{b^{2}} \mp \frac{Q_{e}^{2}}{r^{2} b^{2}}\right) d t^{2}+\left(1-\frac{r^{2}}{b^{2}} \mp \frac{Q_{e}^{2}}{r^{2} b^{2}}\right)^{-1} d r^{2} \\
& +2 d r d t-\frac{2 p}{b}(d t+d r) \sin ^{2} \theta d \phi \\
& +\left(\rho_{+}^{2} \mp N_{m}^{2}\right) d \Omega^{2} .
\end{aligned}
$$

Interestingly Weyl scaling of the metric may be seen to describe an ultra-high energy domain on a specified brane window. The scaling incorporates a change in signature of time in de Sitter geometries. A signature change ensures a temperature and presumably describes a hot de Sitter stringy vacuum [16]. The parameter ' $b$ ' in this regime may be identified with a cosmological scale in the formalism. The difference between an ultra-high and a high energy scale incorporates a small positive vacuum energy density. It elevates the charged quantum black holes to the de Sitter stringy vacua non-perturbatively. The limit $\lambda \rightarrow 1$, underlying the Big Bang singularity, enforces $Q_{e} \rightarrow 0$ at the creation of the brane universe. In a global scenario, i.e. on a pair of $(D \bar{D})_{3}$ branes, the quantum geometries in this regime with real spin angular momenta may be reduced to yield

$$
\begin{aligned}
d s^{2}= & -\left(1-\frac{r^{2}}{b^{2}} \mp \frac{N_{e}^{2}}{r^{2}}\right) d t^{2}+\left(1-\frac{r^{2}}{b^{2}} \mp \frac{N_{e}^{2}}{r^{2}}\right)^{-1} d r^{2} \\
& -\frac{2 p}{b} \sin ^{2} \theta d \phi d t+\left(\rho_{+}^{2} \mp N_{m}^{2}\right) d \Omega^{2} .
\end{aligned}
$$

The conserved electric and magnetic non-linear charges are, respectively, redefined as the point charges $N_{e}=Q_{e} / b$ and $N_{m}=Q_{m} / b$. Then the quantum regime may be re-expressed as $b>r>N_{e}$. A small $N_{e}$ or $N_{m}$ leading to a point charge is ensured by a cosmological scale $b>\left(Q_{e}, Q_{m}\right)$. In the absence of electric and magnetic point charges, the geometries reduce to a de Sitter with a real time. In the presence of charges $\left(N_{e}\right.$ and $\left.N_{m}\right)$ they may be seen to describe a Schwarzschild-de Sitter and a topological de Sitter black hole in Lorentzian signature with a real angular momentum $\Omega^{\phi}$. An $S_{2}$-symmetric geometric patch associated with a magnetic charge appears to decouple from the quantum geometries. However, in its presence the quantum black holes may be re-expressed via a de Sitter vacuum sourced by a two form and a non-perturbative quantum correction sourced by an electric and a magnetic charge. Explicitly they are given by

$$
\begin{aligned}
d s^{2}= & -\left(1-\frac{r^{2}}{b^{2}}\right) d t^{2}+\left(1-\frac{r^{2}}{b^{2}}\right)^{-1} d r^{2} \\
& -\frac{2 p}{b} \sin ^{2} \theta d \phi d t+\rho_{+}^{2} d \Omega^{2} \pm \frac{N_{e}^{2}}{r^{2}} d s_{\mathrm{flat}}^{2}
\end{aligned}
$$

where $d s_{\text {flat }}^{2}=\left(d t^{2}+d r^{2}+r^{2} d \Omega^{2}\right)$ and $Q_{m}=i Q_{e}$. In the absence of $B_{\theta \phi}$ fluctuation, i.e. $p=0$, the two form ansatz (17) primarily accounts for the causal patches of a pure de Sitter in $4 D$ with a cosmological horizon at $r \rightarrow r_{c}=b$. Generically the ansatz $(p \neq 0)$ sources an angular velocity $\Omega^{\phi}$ which incorporates a spin angular momentum in the quantum de Sitter. The hot de Sitter vacua in Eqs. (24) and (25) may be analyzed for the Big Bang singularity in a limit $Q_{e} \rightarrow 0$. The effective de Sitter metric becomes singular there. Thus a pair of $(D \bar{D})_{3}$-branes was vacuum created with non-trivial torsion geometries at a cosmological horizon with the Big Bang. A priori the Riemannian curvature scalar is worked out for the brane/anti-brane together in a limit $Q_{e} \rightarrow 0$ to yield

$$
R=\left(\frac{34}{b^{2}}-\frac{16}{r^{2}}+\frac{2 b^{2}}{r^{4}}\right)
$$

At the cosmological horizon $r \rightarrow b$ the curvature reduces to a small constant value, $R=20 / b^{2}$. It may imply that the brane universe began with the Big Bang. It was described by a small positive cosmological constant and hence may lead to a de Sitter vacuum. Right after the Big Bang the torsion in five dimensions played a significant role to source a one form on a $D_{3}$-brane. It is important to note that an electric non-linear charge, underlying a one form, couples to a flat metric in Eq. (27). It may be identified with a non-perturbative quantum correction presumably to Einstein vacuum in the formalism.

\subsection{Schwarzschild and topological de Sitter}

We analyze the effective de Sitter black holes in Eq. (26) in this section. They describe a Schwazschild de Sitter and a topological de Sitter geometries and are, respectively, given by

$$
\begin{aligned}
d s_{\mathrm{QdS}}^{2}= & -\left(1-\frac{r^{2}}{b^{2}}-\frac{N_{e}^{2}}{r^{2}}\right) d t^{2}-\frac{2 p}{b} \sin ^{2} \theta d \phi d t \\
& +\left(1-\frac{r^{2}}{b^{2}}-\frac{N_{e}^{2}}{r^{2}}\right)^{-1} d r^{2}+\left(\rho_{+}^{2}-N_{m}^{2}\right) d \Omega^{2}
\end{aligned}
$$




$$
\begin{aligned}
d s_{\mathrm{QdT}}^{2}= & -\left(1-\frac{r^{2}}{b^{2}}+\frac{N_{e}^{2}}{r^{2}}\right) d t^{2}-\frac{2 p}{b} \sin ^{2} \theta d \phi d t \\
& +\left(1-\frac{r^{2}}{b^{2}}+\frac{N_{e}^{2}}{r^{2}}\right)^{-1} d r^{2}+\left(\rho_{+}^{2}+N_{m}^{2}\right) d \Omega^{2}
\end{aligned}
$$

The $4 D$ causal de Sitter patches ensure an extra (fifth) dimension between a pair of $(D \bar{D})_{3}$-branes in the formalism. In particular a Schwarzschild dS quantum black hole is characterized by two horizons at

$r_{ \pm}=\frac{r_{c}}{\sqrt{2}}\left(1 \pm \sqrt{1-\frac{4 Q_{e}^{2}}{b^{4}}}\right)^{1 / 2}$.

In this regime the horizon radii are reduced to yield

$r_{+} \approx r_{c}\left(1-\frac{N_{e}^{2}}{2 r_{c}^{2}}\right)$ and $r_{-} \approx N_{e}$.

The topological dS quantum black hole is characterized by an event horizon at

$$
\begin{aligned}
r_{e} & =\frac{r_{c}}{\sqrt{2}}\left(1+\sqrt{1+\frac{4 Q_{e}^{2}}{b^{4}}}\right)^{1 / 2} . \\
& \approx r_{c}\left(1+\frac{N_{e}^{2}}{2 r_{c}^{2}}\right)
\end{aligned}
$$

Thus the event horizons in a Schwarzschild dS and in a topological dS quantum black hole, respectively, can be approximated by $\left(r_{c}-\epsilon\right)$ and $\left(r_{c}+\epsilon\right)$, where $\epsilon<1$. However, their radii in $S_{2}$ appear to shrink or expand in the presence of a two form fluctuation and a magnetic charge. The event horizon is separated from a cosmological horizon by a timelike separation in a Schwarzschild dS quantum black hole. The real spin (angular momentum) turns out to be sourced by an electric charge in the de Sitter black holes. The angular velocities are computed at the event horizon(s) and are given by

$\Omega_{\mathrm{QdS}}^{\phi}=\frac{-b p}{\left(b^{2}-N_{e}^{2}\right)^{2}+\left(p^{2} \sin ^{2} \theta-Q_{m}^{2}\right)}$

and $\Omega_{\mathrm{QdT}}^{\phi}=\frac{-b p}{\left(b^{2}+N_{e}^{2}\right)^{2}+\left(p^{2} \sin ^{2} \theta+Q_{m}^{2}\right)}$.

It ensures a higher spin in a quantum Schwarzschild-de Sitter than in a topological de Sitter black hole. A priori the angular velocities appear to vary with the polar angle, which is sourced by a two form fluctuation $B_{\theta \phi}$ in the formalism. At the creation of a pair of $(D \bar{D})_{3}$-branes, i.e. at the poles, the two form fluctuation vanishes and the angular velocities turn out to be maximum. The spins reduce to a minimum on the equator. The quantum de Sitter black holes presumably enforce a slicing in the polar angle, which may be a consequence of quantized space. The spinning de Sitter quantum black holes do not describe an ergosphere though they are characterized by a conserved angular momentum $p$. However, their radial coordinate is modified $r \rightarrow r_{\text {eff }}$ in the presence of the conserved charges $\left(p, Q_{m}\right)$. They reflect the characteristics of a charged $4 D$ black hole in string theory [43]. In addition the effective radii in the quantum de Sitter black holes in Eqs. (29) and (30) vary with a polar angle and hence deform a sphere to an ellipsoid. The radii increase from their minimum values at the poles to the maximum value on the equator. Formally an effective radius on the equator may be identified with an ergoradius for a stringy Schwarzschild or topological black hole. It may be checked under an interchange $d t_{e} \leftrightarrow d \theta$ and/or $d t_{e} \leftrightarrow d \phi$ with appropriate normalizations in de Sitter quantum black holes. In other words a stringy de Sitter/topological black hole undergoes an expansion from poles to the equator.

In a near (cosmological) horizon regime the $S_{2}$ radii may be approximated to the original radial coordinate $r$. In the limit the quantum black holes (29) and (30) may be approximated by their near horizon geometries. Incorporating a plausible flip in the light cone in the near horizon de Sitter black holes, we obtain

$$
\begin{aligned}
d s_{\mathrm{QdS}}^{2} \rightarrow & -\frac{r^{2}}{N_{e}^{2}} d t^{2}+\frac{N_{e}^{2}}{r^{2}} d r^{2}-\frac{2 p}{b} \sin ^{2} \theta d \phi d t \\
& +r^{2} d \Omega^{2} \\
\text { and } \quad d s_{\mathrm{QdT}}^{2} \rightarrow & -\frac{r^{2}}{N_{m}^{2}} d t^{2}+\frac{N_{m}^{2}}{r^{2}} d r^{2}+r^{2} d \Omega^{2} \\
& -\frac{2 p}{b} \sin ^{2} \theta d \phi d t .
\end{aligned}
$$

It may imply that a Schwarzschild and a topological de Sitter quantum black hole in the near (cosmological) horizon may be viewed through an asymptotic rotating AdS-brane defined, respectively, with the $\mathrm{AdS}$ radii $N_{e}$ and $N_{m}$. The correspondences between an asymptotic AdS quantum black holes and the near (cosmological) horizon Schwarzschild and topological black holes may enforce quantum tunneling: $\mathrm{dS} \rightarrow \mathrm{AdS}$ in the formalism. In fact, tunneling has been argued from a Schwarzschild-de Sitter quantum black hole to a Schwarzschild AdS via a topological de Sitter [16].

A priori an $S_{2}$-symmetric patch associated with a magnetic (non-linear) charge decouples from the stringy black holes. However, we begin with the dyonic charge quantum black holes and work out the a special case for $p \sin \theta= \pm Q_{m}$. Formally, with an euclidean time, they may be given by

$$
\begin{aligned}
d s_{\mathrm{QdS}}^{2}= & \left(1-\frac{r^{2}}{b^{2}}-\frac{N_{e}^{2}}{r^{2}}\right) d t_{e}^{2}+\left(1-\frac{r^{2}}{b^{2}}-\frac{N_{e}^{2}}{r^{2}}\right)^{-1} d r^{2} \\
& \mp 2 N_{m} \sin \theta d \phi d t+\frac{r^{4}}{b^{2}} d \Omega^{2},
\end{aligned}
$$




$$
\begin{aligned}
d s_{\mathrm{QdT}}^{2}= & \left(1-\frac{r^{2}}{b^{2}}+\frac{N_{e}^{2}}{r^{2}}\right) d t_{e}^{2}+\left(1-\frac{r^{2}}{b^{2}}+\frac{N_{e}^{2}}{r^{2}}\right)^{-1} d r^{2} \\
& \mp 2 N_{m} \sin \theta d \phi d t+\left(\frac{r^{4}}{b^{2}}+2 N_{m}^{2}\right) d \Omega^{2} .
\end{aligned}
$$

Since $N_{m}^{2} d \Omega^{2}$ decouples from the rest of the de Sitter topological quantum black hole, the two geometries differ in their sign in the potential sourced by a small electric charge $N_{e}$ in the formalism. With a Lorentzian signature the angular velocity $\Omega^{\phi}$ is real and the quantum black holes are given by

$$
\begin{aligned}
d s_{\mathrm{QdS}}^{2}= & -\left(1-\frac{r^{2}}{b^{2}}-\frac{N_{e}^{2}}{r^{2}}\right) d t^{2} \pm 2 N_{e} \sin \theta d \phi d t \\
& +\left(1-\frac{r^{2}}{b^{2}}-\frac{N_{e}^{2}}{r^{2}}\right)^{-1} d r^{2}+\frac{r^{4}}{b^{2}} d \Omega^{2}, \\
d s_{\mathrm{QdT}}^{2}= & -\left(1-\frac{r^{2}}{b^{2}}+\frac{N_{e}^{2}}{r^{2}}\right) d t^{2} \pm 2 N_{e} \sin \theta d \phi d t \\
& +\left(1-\frac{r^{2}}{b^{2}}+\frac{N_{e}^{2}}{r^{2}}\right)^{-1} d r^{2}+\frac{r^{4}}{b^{2}} d \Omega^{2} .
\end{aligned}
$$

In addition to a cosmological horizon the Schwarzschild dS quantum black hole in Eq. (39) is characterized by an event horizon at $r \rightarrow r_{e}$. The real spin angular momentum turns out to be sourced by an electric charge in the de Sitter black holes. The angular velocities are computed at the event horizon and are given by

$$
\Omega_{\mathrm{QdS}}^{\phi}=\frac{ \pm b^{2} N_{e}}{\left(b^{2}-N_{e}^{2}\right)^{2} \sin \theta}
$$

and $\Omega_{\mathrm{QdT}}^{\phi}=\frac{ \pm b^{2} N_{e}}{\left(b^{2}+N_{e}^{2}\right)^{2} \sin \theta}$.

It ensures a higher spin for a quantum Schwarzschild-de Sitter than a topological de Sitter black hole. A priori the angular velocities appear to vary with a polar angle. It is infinite at poles and takes a minimum value at an equator. The quantum de Sitter black holes presumably enforce a slicing in the polar angle. Nevertheless the need for the $\theta$-slicing geometry may be resolved in the presence of an auxiliary parameter $p$. Then the angular velocities may be re-expressed as

$$
\Omega_{\mathrm{QdS}}^{\phi}=\frac{b p}{\left(b^{2}-N_{e}^{2}\right)^{2}}
$$

and $\Omega_{\mathrm{QdT}}^{\phi}=\frac{b p}{\left(b^{2}+N_{e}^{2}\right)^{2}}$.

For $Q_{m}=0$ the generic quantum black holes (29) and (30) may be simplified for the effective radial coordinate: $r_{\text {eff }}=\left(\sqrt{r^{4}+p^{2} \sin \theta}\right) / b$. For a fixed $r$, it varies from a minimum $r^{2} / b$ to a maximum $\left(\sqrt{r^{4}+p^{2}}\right) / b$. In the limit $r_{\text {eff }} \rightarrow 0$ the de Sitter quantum black holes shrink to a point singularity. Hence a curvature singularity at $r \rightarrow 0$ is removed by a two form fluctuation $(p \neq 0)$ in the de Sitter stringy black holes. Sim- ilarly for $Q_{m} \neq 0$ and $p=0$ a Schwarzschild and a topological de Sitter stringy black hole are, respectively, described by a constant sphere of radii $\left(\sqrt{r^{4}-Q_{m}^{2}}\right) / b$ and $\left(\sqrt{r^{4}-Q_{e}^{2}}\right) / b$. The curvature singularity at $r \rightarrow 0$, in a Schwarzschild-de Sitter quantum and in a topological black hole is removed, respectively, by $r \rightarrow \sqrt{Q_{m}}$ and $r \rightarrow \sqrt{Q_{e}}$.

\subsection{Near horizon geometries: limit $\lambda \rightarrow \mathbf{1}$}

The effective de Sitter geometries, underlying a pair of $(D \bar{D})_{3}$-branes, may be analyzed in the limit $\lambda \rightarrow 1$ to address the origin of the brane universe with the Big Bang. The limit implies $Q_{e} \rightarrow 0$. It does not a priori affect $Q_{m}$ and $p$ associated with the angular coordinates. However, a change in signature underlying the Weyl scaling would imply $Q_{m}=0$ in the de Sitter vacuum at the Big Bang. In addition the angular velocity becomes unphysical with an euclidean signature. It decouples from the de Sitter case in the limit $r \rightarrow b$. In the limit $\lambda \rightarrow 1$ the de Sitter near cosmological horizon geometry (26) may be identified with the near horizon geometry of a quantum black hole (20) on a pair of a brane and an antibrane. In a global scenario the de Sitter vacua immediately after the Big Bang may be approximated for $N_{e}=0$ and $N_{m} \neq 0$. They are reduced to yield

$$
d s^{2}=\left(1-\frac{r^{2}}{b^{2}}\right) d t^{2}+\left(1-\frac{r^{2}}{b^{2}}\right)^{-1} d r^{2}+\rho_{m}^{2} d \Omega^{2},
$$

where the effective radius is

$\rho_{m}=\frac{1}{b} \sqrt{r^{4}+Q_{m}^{2}}$.

A magnetic charge increases the radius of $S_{2}$ in a pure de Sitter vacuum. The de Sitter metric determinant is given by

$$
\begin{aligned}
G & =\frac{r^{8}}{b^{4}}\left(1+\frac{Q_{m}^{2}}{r^{4}}\right)^{2} \sin ^{2} \theta \\
& =\rho_{m}^{4} \sin ^{2} \theta .
\end{aligned}
$$

The metric and the curvature are non-singular in a limit $r \rightarrow b$. However, for $Q_{m}=0$ the de Sitter vacua possess a curvature singularity in the limit of a cosmological horizon and that is interpreted as the Big Bang singularity there. On the other hand the black hole (20) underlying a pre-Weyl scaling under an identical parameterization $\left(Q_{e}=0\right.$ and $\left.p=0\right)$ may be given by

$$
\begin{aligned}
d s^{2}= & -\left(1-\frac{M^{2}}{r^{2}}\right) d t^{2}+\left(1-\frac{M^{2}}{r^{2}}\right)^{-1} d r^{2} \\
& +\frac{M^{2}}{r^{2}} \rho_{m}^{2} d \Omega^{2} .
\end{aligned}
$$

The black hole metric determinant in this case becomes 


$$
\begin{aligned}
\tilde{G} & =-\left(1+\frac{Q_{m}^{2}}{r^{4}}\right) r^{4} \sin ^{2} \theta \\
& =-\frac{b^{4}}{r^{4}} \rho_{m}^{4} \sin ^{2} \theta \\
& =-\lambda^{2} G .
\end{aligned}
$$

Formally a quantum black hole in a Lorentzian signature and a de Sitter vacuum in euclidean signature may be seen to differ by a conformal factor $\lambda(r)$. The black hole is characterized by an event horizon at $r \rightarrow r_{e}=M$. Similar to a de Sitter vacuum the black hole metric and curvature are non-singular in the limit. It possesses a curvature singularity at $r \rightarrow 0$. However, a curvature singularity is ruled out for a definite conformal scale $\lambda(r)$. In the limit $\lambda \rightarrow 1$ the difference in energy scale disappears. In this limit a black hole and a de Sitter vacuum, respectively, approach an event horizon and a cosmological horizon. Formally a coincident horizon at $r_{c}=r_{e}$, underlying two distinct vacua, may lead us to believe that the future time is presumably on a large $S^{1}$. In other words the de Sitter case in a non-perturbative formulation of quantum gravity may alternately be viewed through an exact Schwarzschild underlying a macroscopic black hole in Einstein gravity. Thus a thermal de Sitter (a small time) and a large time black hole may be related by $T$-duality underlying $R \rightarrow \alpha^{\prime} / R$ in string theory. It is further supported by the fact that a $4 D$ thermal de Sitter quantum vacuum is conformally identified with a Lorentzian Schwarzschild black hole sourced by a $5 \mathrm{D}$ metric potential in the formalism.

\section{Torsion geometries in 4D}

In this section we focus on a generalized brane geometry underlying the dynamical geometric torsion in the formalism. We obtain torsion geometries leading to a generalized $4 D$ Reissner-Nordstrom ( $\mathrm{RN}$ ) black hole which is essentially sourced by the zero and non-zero modes of a two form. The torsion potential may be seen to describe the high energy (stringy) modes and hence they may be ignored in the low energy limit. The generalized (quantum) black hole in the limit shall be shown to reduce to an RN vacuum in Einstein gravity.

In this case we consider the ansatz

$$
\begin{aligned}
B_{t \theta} & =B_{r \theta}=b \\
\text { and } \quad B_{t \phi} & =-M^{2} \cos \theta .
\end{aligned}
$$

Then the non-trivial components of a geometric torsion are worked out:

$$
\begin{aligned}
\mathcal{H}_{t \theta \phi} & \rightarrow H_{t \theta \phi}=-M^{2} \sin \theta \\
\text { and } \mathcal{H}_{t r \phi} & =\frac{M^{2} b}{r^{2}} \sin \theta .
\end{aligned}
$$

The geometric torsion incorporates metric fluctuations into the flat vacuum on a brane. Then the emergent metric on a vacuum created pair of $(D \bar{D})_{3}$-branes is sourced by the global modes of a NS two form in addition to the local modes of a two form in a non-linear $U(1)$ gauge theory. It is given by

$G_{\mu \nu}=g_{\mu \nu} \pm B_{\mu \lambda} g^{\lambda \rho} B_{\rho \nu}+C \overline{\mathcal{H}}_{\mu \lambda \rho} g^{\lambda \alpha} g^{\rho \beta} \overline{\mathcal{H}}_{\alpha \beta \nu}$.

For $C=( \pm 1 / 2)$ the line element is given by

$$
\begin{aligned}
d s^{2}= & -\left(1 \pm \frac{b^{2}}{r^{2}} \pm \frac{M^{4}}{r^{4}} \pm \frac{M^{4} b^{2}}{r^{6}}\right) d t^{2} \mp \frac{2 b^{2}}{r^{2}} d t d r \\
& +\left(1 \mp \frac{b^{2}}{r^{2}} \mp \frac{M^{4} b^{2}}{r^{6}}\right) d r^{2}+\left(1 \mp \frac{M^{4}}{r^{4}}\right) r^{2} d \theta^{2} \\
& +\left(1 \mp \frac{M^{4}}{r^{4}} \mp \frac{M^{4} b^{2}}{r^{6}}\right) r^{2} \sin ^{2} \theta d \phi^{2} \\
& \pm \frac{2 M^{4} b}{r^{4}} d r d \theta
\end{aligned}
$$

\subsection{Stringy RN-like de Sitter}

In this section we analyze the emergent geometries in a brane window for $r>(b, M)$. They shall be seen to describe the quantum Reissner-Nordstrom and Schwarzschild black holes on a pair of $(D \bar{D})_{3}$-branes in the presence of an extra dimension. The quantum patches may be rearranged in this regime on a vacuum created $D_{3}$-brane with $r^{4}>>b^{4}$ and $r^{8}>>M^{8}$ to yield

$$
\begin{aligned}
d s^{2}= & -\left(1 \pm \frac{b^{2}}{r^{2}} \mp \frac{M^{4}}{r^{4}} \pm \frac{M^{4} b^{2}}{r^{6}}\right) d t^{2} \\
& +\left(1 \pm \frac{b^{2}}{r^{2}} \mp \frac{M^{4}}{r^{4}} \pm \frac{M^{4} b^{2}}{r^{6}}\right)^{-1} d r^{2}+r^{2} d \theta^{2} \\
& +\left(1 \mp \frac{M^{4} b^{2}}{r^{6}}\right) r^{2} \sin ^{2} \theta d \phi^{2} \\
& \mp \frac{2 b^{2}}{r^{2}}\left(d t-\frac{M^{4}}{b r^{2}} d \theta\right) d r \mp \frac{M^{4}}{r^{4}} d s_{\text {flat }}^{2},
\end{aligned}
$$

where $d S_{\text {flat }}^{2}=\left(2 d t^{2}+d r^{2}+r^{2} d \Omega^{2}\right)$.

In fact the non-trivial quantum geometries are essentially sourced by a higher form gauge field or a two form on a $D_{4}$-brane, which in turn describes a vacuum created pair of $(D \bar{D})_{3}$-branes. It forces one to consider a global scenario underlying a pair. The relevant brane geometries on a pair of $(D \bar{D})_{3}$-branes become

$$
\begin{aligned}
d s^{2}= & -\left(1 \pm \frac{b^{2}}{r^{2}}-\frac{M^{4}}{r^{4}}+\frac{M^{4} b^{2}}{r^{6}}\right) d t^{2} \\
& +\left(1 \pm \frac{b^{2}}{r^{2}}-\frac{M^{4}}{r^{4}}+\frac{M^{4} b^{2}}{r^{6}}\right)^{-1} d r^{2}+r^{2} d \theta^{2} \\
& +\left(1-\frac{M^{4} b^{2}}{r^{6}}\right) r^{2} \sin ^{2} \theta d \phi^{2}
\end{aligned}
$$


and

$$
\begin{aligned}
d s^{2}= & -\left(1 \pm \frac{b^{2}}{r^{2}}+\frac{M^{4}}{r^{4}}-\frac{M^{4} b^{2}}{r^{6}}\right) d t^{2} \\
& +\left(1 \pm \frac{b^{2}}{r^{2}}+\frac{M^{4}}{r^{4}}-\frac{M^{4} b^{2}}{r^{6}}\right)^{-1} d r^{2}+r^{2} d \theta^{2} \\
& +\left(1+\frac{M^{4} b^{2}}{r^{6}}\right) r^{2} \sin ^{2} \theta d \phi^{2} .
\end{aligned}
$$

The first deformed term, in the causal patches, is sourced by the zero modes in $B_{2}$-flux. It describes a Schwarzschild metric potential in $4 D$ with an extra dimension. The second and third term correspond to the charges, respectively, underlying an axionic flux and a geometric torsion in the formalism. In addition the spherical symmetry is broken by a geometric torsion in the emergent quantum vacua. It may be recalled that a geometric torsion is primarily sourced by all the higher order terms underlying a $B_{2}$-coupling to an axion in a gauge theory.

\subsection{Non-propagating torsion geometries}

In the absence of a geometric torsion one of the quantum geometries (53) precisely describes a quantum RN black hole in $4 D$ with a hint to a fifth dimension. Explicitly the relevant quantum black hole is given by

$$
\begin{aligned}
d s_{\mathrm{QRN}}^{2}= & -\left(1-\frac{b^{2}}{r^{2}}+\frac{M^{4}}{r^{4}}\right) d t^{2} \\
& +\left(1-\frac{b^{2}}{r^{2}}+\frac{M^{4}}{r^{4}}\right)^{-1} d r^{2}+r^{2} d \Omega^{2} .
\end{aligned}
$$

An extra dimension is intrinsic to a $B_{2}$-flux. It ensures a precise electromagnetic charge, via a Poincaré duality from an axionic charge $M$, in the RN black hole. It shows that constant two form components source a mass term and a local two form sources an electric (magnetic) charge. In a low energy limit the quantum vacuum reduces to a typical RN black hole in Einstein gravity. A formal identification of a quantum RN vacuum with its (semi) classical geometry ensures an exact black hole in the formalism.

A priori three among the four quantum geometries (52)(53), in the absence of geometric torsion, may be seen to reduce to some of the known black holes in the Einstein vacuum. The fourth geometry, under a flip of the light cone at the event horizon, will be seen to identify with one among the remaining three in a perturbative gauge theory. Firstly we focus on two of the relevant vacua and they are given by

$$
\begin{aligned}
d s^{2}= & -\left(1-\frac{b^{2}}{r^{2}} \mp \frac{M^{4}}{r^{4}}\right) d t^{2} \\
& +\left(1-\frac{b^{2}}{r^{2}} \mp \frac{M^{4}}{r^{4}}\right)^{-1} d r^{2}+r^{2} d \Omega^{2} .
\end{aligned}
$$

They may be worked out for their renormalized black hole masses $b_{\text {ren }}$, which formally leads to a 4D quantum Schwarzschild black hole in the presence of an extra dimension in the formalism. It is given by

$$
\begin{aligned}
& d s^{2}=-\left(1-\frac{b_{\text {ren }}^{2}}{r^{2}}\right) d t^{2}+\left(1-\frac{b_{\text {ren }}^{2}}{r^{2}}\right)^{-1} d r^{2}+r^{2} d \Omega^{2} \\
& \quad \text { where } b_{\text {ren }}^{2}=b^{2}\left(1+\frac{N^{2}}{r^{2}}\right)_{r \rightarrow r_{h}=b_{\text {ren }}} \\
& \quad \text { and } N=\frac{M^{2}}{b} .
\end{aligned}
$$

The local degrees in a higher form gauge field are absorbed by the black hole mass to describe a dynamical vacuum. The computation of the renormalized mass, at the event horizon of each black hole, takes three different values $\left(b_{s}, b_{+}\right.$and $b_{-}$). Formally, they are

$b_{\text {ren }}=\frac{b}{\sqrt{2}}\left(1 \pm \sqrt{1+\frac{4 M^{4}}{b^{4}}}\right)^{1 / 2}$.

Explicitly the renormalized mass $b_{1}$ of the Schwarzschild black hole (55) defined with $-M^{4}$ in the $G_{t t}$ component becomes

$b_{s}=\frac{b}{\sqrt{2}}\left(1+\sqrt{1+\frac{4 M^{4}}{b^{4}}}\right)^{1 / 2}$.

The remaining geometry corresponds to a RN black hole (54). It may be re-expressed with two values of renormalized masses and we have

$$
\begin{gathered}
b_{+}=\frac{b}{\sqrt{2}}\left(1+\sqrt{1-\frac{4 M^{4}}{b^{4}}}\right)^{1 / 2} \\
\text { and } b_{-}=\frac{b}{\sqrt{2}}\left(1-\sqrt{1-\frac{4 M^{4}}{b^{4}}}\right)^{1 / 2} .
\end{gathered}
$$

It ensures the black hole horizons at $r \rightarrow r_{ \pm}=b_{ \pm}$. In other words a Reissner-Nordstrom black hole may be interpreted as a multi-black hole solution in the formalism. The relative strength of the parameters $(b, M)$ may further be explored to obtain a simplified expression for the renormalized masses. Within a brane window, i.e. $r>(b, M)$ with $r^{4}>>b^{4}$ and $r^{8}>>M^{8}$, firstly we consider $b=M$. In this case the geometry reduces to a background Schwarzschild black hole defined with an event horizon $b_{\text {ren }}=b_{s} \rightarrow b$. The local degree underlying torsion drops out in this regime. Secondly for $M>b$ and $M^{2}>>b^{2}$ the only sensible renormalized mass may be approximated by $b_{s}=M$ and describes a macroscopic black hole. Thirdly for $M<b$ and $M^{8}<<b^{8}$ the renormalized masses are approximated by $b_{s}=\left(b+M^{4} / 2 b^{3}\right), b_{+}=\left(b-M^{4} / 2 b^{3}\right)$, and $b_{-}=\left(M^{2} / b\right)$ 
and they correspond to a macroscopic Reissner-Nordstrom black hole.

On the other hand the gauge theoretic torsion or its parameter $M$ underlying the axionic charge in $4 D$ may alternately be identified with an electromagnetic charge in the presence of a fifth dimension. A renormalized non-linear charge $M_{\text {ren }}$ is worked out by absorbing a cloud of zero modes of $B_{2}$-flux in its non-zero modes. In the absence of the geometric torsion the brane geometry (52) defined with $+b^{2} / r^{2}$ in the $G_{t t}$ component may be re-expressed as

$$
\begin{aligned}
d s^{2}= & -\left(1-\frac{M_{\mathrm{ren}}^{2}}{r^{4}}\right) d t^{2}+\left(1-\frac{M_{\mathrm{ren}}^{2}}{r^{4}}\right)^{-1} d r^{2} \\
& +r^{2} d \Omega^{2}
\end{aligned}
$$

$$
\text { where } M_{\text {ren }}=M\left(1-\frac{r^{2}}{N^{2}}\right)_{r \rightarrow r_{h}=M_{\text {ren }}}^{1 / 4} .
$$

The renormalized mass of a Schwarzschild black hole mass (60) may explicitly be worked out:

$M_{\text {ren }}=\frac{b}{\sqrt{2}}\left(-1+\sqrt{1+\frac{4 M^{4}}{b^{4}}}\right)^{1 / 2}$.

Similarly in the absence of a geometric torsion the brane geometry (53) defined with $\left(+b^{2} / r^{2}\right)$ in the $G_{t t}$ component may be identified with a Schwarzschild black hole (56) for its mass (58) under a flip of the light cone at the event horizon. The renormalized black hole mass may be approximated to yield $M_{\text {ren }}=M$ and $M_{\text {ren }}=\left(M^{2} / b\right)$, respectively, for $M>b$ and $\left(M<b\right.$ with $\left.M^{8}<<b^{8}\right)$. Equations (57), (58), and (62) further imply $M_{\text {ren }}<b_{\text {ren. The renormalized masses }}$ leading to the Schwazschild geometries (60), respectively, with mass $M$ and $\left(M^{2} / b\right)$ correspond to a microscopic black hole. It implies that a two form underlying its (renormalized) zero mode may define the mass of a classical Schwarzschild black hole in a non-perturbation geometric torsion formalism. In the low energy limit all the local degrees in a geometric torsion are absorbed in the renormalized mass $b_{\text {ren }}$ to define the Einstein vacuum without any propagating torsion. A renormalized non-zero mode of the two form defines a non-linear $U(1)$ charge in a quantum Schwarzschild black hole underlying the perturbation theory.

Interestingly a renormalized charge is worked out at an event horizon to obtain $M_{\text {ren }}=b_{\text {ren }}$. It establishes the fact that a Schwarzschild black hole is indeed unique. In fact a precise identification of a renormalized charge with a (renormalized) mass of a black hole is an artifact of higher form $U$ (1) gauge theory on a $D_{3}$-brane. Thus a Schwarzschild black hole in the formalism is precisely described in a perturbation gauge theory on a $D_{3}$-brane in the absence of a geometric torsion. In other words the low energy limit ensures a non-propagating torsion in the formalism. As a result the generalized curvature reduces to Riemannian geometry in the limit and is described by a perturbation theory. Then the black hole mass in Eq. (56) ensures a large ' $M$ ' and a small ' $b$ ' for a typical Schwarzschild black hole. In fact a large and a small value of the parameters are indeed enforced by the underlying $U(1)$ gauge theory in $H_{3}$ defined with a small $B_{2}$ coupling (5). A priori the emergent Schwarzschild black hole (60) defined with $M_{\text {ren }}$ hints at three extra dimensions unlike the one extra dimension with $b_{\text {ren }}$ in Eq. (56). However, an analysis underlying a zero and a non-zero mode of the $B_{2}$ flux reveals a fifth extra dimension. It resolves the apparent disparity in the number of extra dimensions between two alternate descriptions.

\subsection{Stringy de Sitter black holes}

Now we Weyl scale the emergent quantum geometries by $\lambda=\left(b^{2} / r^{2}\right)$ on a $D_{3}$-brane. Under the $\lambda$ scaling the quantum geometric patches sourced by the geometric torsion in the formalism may be described by

$$
\begin{aligned}
d s^{2}= & \left(1-\frac{r^{2}}{b^{2}} \pm \frac{N^{2}}{r^{2}} \mp \frac{M^{4}}{r^{4}}\right) d t^{2}+2 d t d r+\frac{r^{4}}{b^{2}} d \Omega^{2} \\
& +\left(1+\frac{r^{2}}{b^{2}} \pm \frac{N^{2}}{r^{2}} \mp \frac{M^{4}}{r^{4}}\right) d r^{2} \mp \frac{M^{4}}{r^{2}} \sin ^{2} \theta d \phi^{2} \\
& \mp \frac{N^{2}}{r^{2}}\left(2 d t^{2}+d r^{2}+r^{2} d \Omega^{2}\right) \pm \frac{2 M^{4}}{b r^{2}} d r d \theta
\end{aligned}
$$

and $d s^{2}=-\left(1+\frac{r^{2}}{b^{2}} \mp \frac{N^{2}}{r^{2}} \pm \frac{M^{4}}{r^{4}}\right) d t^{2}-2 d t d r$

$$
\begin{aligned}
& -\left(1-\frac{r^{2}}{b^{2}} \mp \frac{N^{2}}{r^{2}} \pm \frac{M^{4}}{r^{4}}\right) d r^{2}+\frac{r^{4}}{b^{2}} d \Omega^{2} \\
& \pm \frac{M^{4}}{b r^{2}} d r d \theta \mp \frac{M^{4}}{r^{2}} \sin ^{2} \theta d \phi^{2} \\
& \mp \frac{N^{2}}{r^{2}}\left(2 d t^{2}+d r^{2}+r^{2} d \Omega^{2}\right) .
\end{aligned}
$$

We investigate the quantum regime for $M<r<b$ on a $D_{3}$-brane. Then the metric component $G_{r r}$ may be approximated with $M^{8}<<r^{8}$ and $r^{4}<<b^{4}$. They may a priori be described by some of the AdS and de Sitter causal patches on the $D_{3}$-brane. However, the causal patches, sourced by the local modes of a higher form, appear to be associated with a wrong relative sign. They are given by

$$
\begin{aligned}
d s^{2}=( & \left.1-\frac{r^{2}}{b^{2}} \pm \frac{N^{2}}{r^{2}} \mp \frac{M^{4}}{r^{4}}\right) d t^{2}+2 d t d r+\frac{r^{4}}{b^{2}} d \Omega^{2} \\
& +\left(1-\frac{r^{2}}{b^{2}} \mp \frac{N^{2}}{r^{2}} \pm \frac{M^{4}}{r^{4}}\right)^{-1} d r^{2} \pm \frac{2 M^{4}}{b r^{2}} d r d \theta \\
& \mp \frac{N^{2}}{r^{2}}\left(2 d t^{2}+d r^{2}+r^{2} d \Omega^{2}\right) \mp \frac{M^{4}}{r^{2}} \sin ^{2} \theta d \phi^{2}
\end{aligned}
$$




$$
\text { and } \begin{aligned}
d s^{2}= & -\left(1+\frac{r^{2}}{b^{2}} \mp \frac{N^{2}}{r^{2}} \pm \frac{M^{4}}{r^{4}}\right) d t^{2}-2 d t d r \\
& -\left(1+\frac{r^{2}}{b^{2}} \pm \frac{N^{2}}{r^{2}} \mp \frac{M^{4}}{r^{4}}\right)^{-1} d r^{2} \\
& \mp \frac{M^{4}}{r^{2}} \sin ^{2} \theta d \phi^{2} \pm \frac{2 M^{4}}{b r^{2}} d r d \theta+\frac{r^{4}}{b^{2}} d \Omega^{2} \\
& \mp \frac{N^{2}}{r^{2}}\left(2 d t^{2}+d r^{2}+r^{2} d \Omega^{2}\right) .
\end{aligned}
$$

Under the interchange $d t_{e} \leftrightarrow d r$, the naive AdS causal patches (65) may be rearranged to describe the de Sitter vacua (64). In a global scenario, i.e. on a pair of vacuum created $(D \bar{D})_{3}$-branes, the quantum geometries may be re-expressed as

$$
\begin{aligned}
d s^{2}= & \left(1-\frac{r^{2}}{b^{2}} \mp \frac{N^{2}}{r^{2}} \pm \frac{M^{4}}{r^{4}}\right) d t^{2}+\frac{r^{4}}{b^{2}} d \Omega^{2} \\
& +\left(1-\frac{r^{2}}{b^{2}} \pm \frac{N^{2}}{r^{2}} \mp \frac{M^{4}}{r^{4}}\right)^{-1} d r^{2} \\
& \mp \frac{M^{4}}{r^{2}} \sin ^{2} \theta d \phi^{2} \mp \frac{N^{2}}{r^{2}} d s_{\text {flat }}^{2} .
\end{aligned}
$$

On the other hand the causal patches in the emergent quantum geometries may be viewed through a $(2 \times 2)$ matrix $\mathcal{M}$ projection [16] on the column vectors:

$$
\left(\begin{array}{l}
1 \\
0
\end{array}\right) \text { and }\left(\begin{array}{l}
0 \\
1
\end{array}\right) \text {. }
$$

The causal patches define a matrix, given by

$$
\mathcal{M}=\frac{1}{2}\left(\begin{array}{cc}
\tilde{G}_{t t}^{\prime}(+) & \tilde{G}_{r r}^{\prime}(+) \\
\tilde{G}_{r r}^{\prime}(-) & \tilde{G}_{t t}^{\prime}(-)
\end{array}\right),
$$

where $\quad \tilde{G}_{t t}^{\prime}(+)=\left(1-\frac{r^{2}}{b^{2}}+\frac{N^{2}}{r^{2}}-\frac{M^{4}}{r^{4}}\right)$,

$$
\begin{aligned}
\tilde{G}_{r r}^{\prime}(+) & =\left(1-\frac{r^{2}}{b^{2}}+\frac{N^{2}}{r^{2}}-\frac{M^{4}}{r^{4}}\right)^{-1}, \\
\tilde{G}_{t t}^{\prime}(-) & =\left(1-\frac{r^{2}}{b^{2}}-\frac{N^{2}}{r^{2}}+\frac{M^{4}}{r^{4}}\right) \\
\text { and } \quad \tilde{G}_{r r}^{\prime}(-) & =\left(1-\frac{r^{2}}{b^{2}}-\frac{N^{2}}{r^{2}}+\frac{M^{4}}{r^{4}}\right)^{-1} .
\end{aligned}
$$

The inverse of the matrix becomes

$$
\mathcal{M}^{-1}=\frac{1}{2 \operatorname{det} \mathcal{M}}\left(\begin{array}{cc}
\tilde{G}_{t t}^{\prime}(-) & -\tilde{G}_{r r}^{\prime}(+) \\
-\tilde{G}_{r r}^{\prime}(-) & \tilde{G}_{t t}^{\prime}(+)
\end{array}\right) .
$$

It is important to note that the matrix projection on two independent column vectors projects the quantum causal patches obtained on a brane (66). Explicitly they are

$$
\begin{aligned}
\mathcal{M}\left(\begin{array}{l}
1 \\
0
\end{array}\right) & =\frac{1}{2}\left(\begin{array}{c}
\tilde{G}_{t t}^{\prime}(+) \\
\tilde{G}_{r r}^{\prime}(-)
\end{array}\right) \\
\text { and } \mathcal{M}\left(\begin{array}{l}
0 \\
1
\end{array}\right) & =\frac{1}{2}\left(\begin{array}{c}
\tilde{G}_{r r}^{\prime}(+) \\
\tilde{G}_{t t}^{\prime}(-)
\end{array}\right) .
\end{aligned}
$$

The determinant of the matrix is worked out in the brane regime to yield

$\operatorname{det} \mathcal{M}=-\frac{r^{2}}{b^{2}}$.

The determinant at the cosmological horizon ensures (det $\mathcal{M}=-1)$ a discrete transformation, underlying a nondegenerate matrix $\mathcal{M}$ for its projection on two dimensional column vectors. We perform an inverse matrix operation on the column vectors to separate out the mixed causal patches in the quantum regime. They are given by

$\mathcal{M}^{-1}\left(\begin{array}{l}1 \\ 0\end{array}\right)=\frac{1}{2}\left(\begin{array}{c}-\tilde{G}_{t t}^{\prime}(-) \\ \tilde{G}_{r r}^{\prime}(-)\end{array}\right)$

and

$\mathcal{M}^{-1}\left(\begin{array}{l}0 \\ 1\end{array}\right)=\frac{1}{2}\left(\begin{array}{c}\tilde{G}_{r r}^{\prime}(+) \\ -\tilde{G}_{t t}^{\prime}(+)\end{array}\right)$.

Then the inverse matrix projected quantum causal patches on a brane may appropriately be re-expressed as

$$
\begin{aligned}
d s^{2}= & -\left(1-\frac{r^{2}}{b^{2}}-\frac{N^{2}}{r^{2}}+\frac{M^{4}}{r^{4}}\right) d t^{2}-\frac{M^{4}}{r^{2}} \sin ^{2} \theta d \phi^{2} \\
& +\left(1-\frac{r^{2}}{b^{2}}-\frac{N^{2}}{r^{2}}+\frac{M^{4}}{r^{4}}\right)^{-1} d r^{2}+\frac{r^{4}}{b^{2}} d \Omega^{2} \\
+ & \frac{N^{2}}{r^{2}} d s_{\text {flat }}^{2} \\
\text { and } d s^{2}= & -\left(1-\frac{r^{2}}{b^{2}}+\frac{N^{2}}{r^{2}}-\frac{M^{4}}{r^{4}}\right) d t^{2}+\frac{r^{4}}{b^{2}} d \Omega^{2} \\
& +\left(1-\frac{r^{2}}{b^{2}}+\frac{N^{2}}{r^{2}}-\frac{M^{4}}{r^{4}}\right)^{-1} d r^{2} \\
& +\frac{M^{4}}{r^{2}} \sin ^{2} \theta d \phi^{2}-\frac{N^{2}}{r^{2}} d s_{\text {flat }}^{2} .
\end{aligned}
$$

A priori the causal patches reveal a quantum ReissnerNordstrom de Sitter (73) and a quantum Schwarzschild-de Sitter (74) geometry. In this case the defined parameter $N$ may be interpreted as the mass of a black hole and $M$ defines a two form non-linear charge. However, two among the three parameters $(b, N, M)$ are independent. Thus the apparent Reissner-Nordstrom de Sitter vacuum is indeed an artifact 
of the naive parameterization. In the absence of the local degrees in a torsion, i.e. for $M=0$, the emergent geometries reduce to a spherically symmetric de Sitter vacuum in 4D. Ignoring the flat metric term in the quantum geometries the non-trivial de Sitter vacua (73) and (74) may be given by

$$
\begin{aligned}
d s^{2}= & -\left(1-\frac{r^{2}}{b^{2}}\right)\left(1+\frac{M^{4}}{r^{4}}\right) d t^{2}+\frac{r^{4}}{b^{2}} d \theta^{2} \\
& +\left(1-\frac{r^{2}}{b^{2}}\right)^{-1}\left(1+\frac{M^{4}}{r^{4}}\right)^{-1} d r^{2} \\
& +\left(\frac{r^{2}}{b^{2}}-\frac{M^{4}}{r^{4}}\right) r^{2} \sin ^{2} \theta d \phi^{2},
\end{aligned}
$$

$$
\text { and } \begin{aligned}
d s^{2}= & -\left(1-\frac{r^{2}}{b^{2}}\right)\left(1-\frac{M^{4}}{r^{4}}\right) d t^{2}+\frac{r^{4}}{b^{2}} d \theta^{2} \\
& +\left(1-\frac{r^{2}}{b^{2}}\right)^{-1}\left(1-\frac{M^{4}}{r^{4}}\right)^{-1} d r^{2} \\
& +\left(\frac{r^{2}}{b^{2}}+\frac{M^{4}}{r^{4}}\right) r^{2} \sin ^{2} \theta d \phi^{2} .
\end{aligned}
$$

The de Sitter vacuum (75) is defined with a cosmological horizon at $r \rightarrow r_{c}=b$. A local torsion breaks the $S_{2-}$ symmetry and deforms the causal patches without any significant change and hence may be identified with a topological de Sitter black hole in 4D. The other de Sitter vacuum (76) possesses an event horizon at $r \rightarrow r_{e}=M$ in addition to a cosmological horizon at $r_{c}=b$. It describes a Schwarzschild-de Sitter black hole in 4D. Most importantly a superposition of two independent geometries ensures a pure de Sitter vacuum. It is essentially sourced by the zero modes of a two form in the formalism. The cancelation of the nonzero modes' contribution or the local degrees of a geometric torsion in the overlapping geometry ensures a gauge theoretic vacuum on a $D_{4}$-brane. In other words a topological de Sitter and a Schwarzschild-de Sitter quantum black holes are created at the Big Bang singularity $r_{c}=b$, respectively, on a $D_{3}$-brane and a $\bar{D}_{3}$-brane. The black hole geometries are indeed sourced by the geometric torsion in the formalism.

The non-trivial section in the quantum de Sitter case, (73), on a brane may also be re-expressed in terms of the renormalized mass $N_{\text {eff }}$ or the charge $M_{\text {eff }}$. It takes the form

$$
\begin{aligned}
d s^{2}= & -\left(1-\frac{r^{2}}{b^{2}}+\frac{N_{\mathrm{eff}}^{2}}{r^{2}}\right) d t^{2}+\left(1-\frac{r^{2}}{b^{2}}+\frac{N_{\mathrm{eff}}^{2}}{r^{2}}\right)^{-1} d r^{2} \\
& +\frac{r^{4}}{b^{2}} d \theta^{2}+\left(\frac{r^{2}}{b^{2}}-\frac{M^{4}}{r^{4}}\right) r^{2} \sin ^{2} \theta d \phi^{2}
\end{aligned}
$$

or

$$
\begin{aligned}
& \begin{aligned}
d s^{2}= & -\left(1-\frac{r^{2}}{b^{2}}+\frac{M_{\mathrm{eff}}^{4}}{r^{4}}\right) d t^{2}+\left(1-\frac{r^{2}}{b^{2}}+\frac{M_{\mathrm{eff}}^{4}}{r^{4}}\right)^{-1} d r^{2} \\
+ & \frac{r^{4}}{b^{2}} d \theta^{2}+\left(\frac{r^{2}}{b^{2}}-\frac{M^{4}}{r^{4}}\right) r^{2} \sin ^{2} \theta d \phi^{2}
\end{aligned} \\
& \text { where } \quad N_{\mathrm{eff}}^{2}=N^{2}\left(\frac{b^{2}}{r_{e}^{2}}-1\right) \\
& \text { and } \quad M_{\mathrm{eff}} \approx M\left(1-\frac{r_{h}^{2}}{4 b^{2}}\right)
\end{aligned}
$$

The causal patches in the quantum geometry (77) may be identified with a four dimensional TdS black hole in the presence of an extra dimension in Einstein vacuum. However, a propagating torsion (48) in the formalism ensures a non-Riemannian geometry underlying a quantum TdS black hole. The Ricci scalar takes the form

$R=\frac{F(M, b, \theta)}{b^{2} r^{6}\left(b^{2} M^{4}-r^{6}\right)}$.

A priori a quantum TdS black hole possesses curvature singularities at $r \rightarrow 0$ and at $r \rightarrow\left(b M^{2}\right)^{1 / 3}$. Nevertheless a non-Riemannian torsion geometry leading to an effective TdS quantum black hole is described by a modified curvature scalar $\mathcal{K}$. The curvature scalar is computed in a TdS quantum black hole which is sourced by a geometric torsion. It implies

$R \rightarrow \mathcal{K}=\frac{M^{4}}{2 r^{4}}\left(1+\frac{b^{2}}{r^{2}}\right)$

Naively a TdS quantum black hole possesses a curvature singularity defined in the limit $r \rightarrow 0$. However, on a brane window $M<r<b$ the geometric torsion forbids the possibility of a curvature singularity in a TdS quantum geometry on a brane. The geometric torsion, leading to a quantum black hole in string theory, presumably manifests a gravitational repulsion that prevents the formation of singularities in the vacuum created brane/anti-brane at the Big Bang. Interestingly, the emergent black hole on a brane is characterized by an event horizon at

$r \rightarrow r_{h} \approx b\left(1+\frac{N_{\mathrm{eff}}^{2}}{2 b^{2}}\right)$.

In the near horizon limit $r \rightarrow b$ the curvature scalar takes the smallest positive constant value and further reconfirms a de Sitter geometry on a vacuum created pair of $(D \bar{D})_{3}$-branes. Similarly the non-trivial line element in the de Sitter black hole (74) may be re-expressed in terms of its renormalized mass or its renormalized charge. It is given by

$$
\begin{aligned}
d s^{2}= & -\left(1-\frac{r^{2}}{b^{2}}-\frac{N_{\text {eff }}^{2}}{r^{2}}\right) d t^{2}+\left(1-\frac{r^{2}}{b^{2}}-\frac{N_{\text {eff }}^{2}}{r^{2}}\right)^{-1} d r^{2} \\
& +\left(\frac{r^{2}}{b^{2}}+\frac{M^{4}}{r^{4}}\right) r^{2} \sin ^{2} \theta d \phi^{2}+\frac{r^{4}}{b^{2}} d \theta^{2}
\end{aligned}
$$


or

$$
\begin{aligned}
d s^{2}= & -\left(1-\frac{r^{2}}{b^{2}}-\frac{M_{\mathrm{eff}}^{4}}{r^{4}}\right) d t^{2}+\left(1-\frac{r^{2}}{b^{2}}-\frac{M_{\mathrm{eff}}^{4}}{r^{4}}\right)^{-1} d r^{2} \\
& +\frac{r^{4}}{b^{2}} d \theta^{2}+\left(\frac{r^{2}}{b^{2}}+\frac{M^{4}}{r^{4}}\right) r^{2} \sin ^{2} \theta d \phi^{2}
\end{aligned}
$$

It describes a four dimensional $\mathrm{SdS}$ quantum geometry sourced by a geometric torsion on a vacuum created pair of $(D \bar{D})_{3}$-branes. Though the curvature singularity is absent in the brane regime, the geometry describes a black hole with a cosmological horizon $r_{c}$ and an event horizon $r_{e}$. Explicitly they may be approximated to yield

$r_{c} \approx b\left(1-\frac{N_{\mathrm{eff}}^{2}}{2 b^{2}}\right) \quad$ and $r_{e} \approx N_{\mathrm{eff}}$.

The horizon radii in the quantum black holes (78) and (84) may also be estimated by their renormalized charge $M_{\text {eff }}$. The emergent TdS and SdS geometries may, respectively, be viewed through their origin in a vacuum created $D_{3}$-brane and an anti- $D_{3}$-brane or vice versa at a cosmological horizon $r_{c}=b$, which was shown to describe the Big Bang singularity in Ref. [16]. In fact it was argued that the brane universe began instantaneously underlying a vacuum created pair of D-instantons by a higher form gauge field on a $D_{4}$ brane. The coupling of a dynamical torsion, i.e. $M \neq 0$, leads to two different quantum vacua underlying a TdS and a SdS black hole. Qualitatively the event horizon in both cases may be described at $r \rightarrow \sqrt{b}$. However, a TdS black hole corresponds to an upper cut-off $b<1$ and a SdS black hole is defined with a lower cut-off $1<b$. In other words, a geometric torsion begins to interplay at $b=1$ and distinguishes a TdS quantum black hole from a SdS quantum black hole. The geometric torsion also breaks the spherical symmetry. The metric potential signifies an extra fifth dimension between a pair. Interestingly the geometric torsion sources a high energy mode and ensures underlying stringy aspects in the emergent TdS and SdS geometries on a non-BPS brane.

In a low energy limit the torsion contribution becomes insignificant in the de Sitter black holes. In this limit a TdS and a SdS black hole are approximated by the Riemannian curvature and are, respectively, given by

$$
\begin{aligned}
d s^{2}= & -\left(1-\frac{r^{2}}{b^{2}}-\frac{N^{2}}{r^{2}}\right) d t^{2}+\left(1-\frac{r^{2}}{b^{2}}-\frac{N^{2}}{r^{2}}\right)^{-1} d r^{2} \\
& +\frac{r^{4}}{b^{2}} d \Omega^{2} \\
d s^{2}= & -\left(1-\frac{r^{2}}{b^{2}}+\frac{N^{2}}{r^{2}}\right) d t^{2}+\left(1-\frac{r^{2}}{b^{2}}+\frac{N^{2}}{r^{2}}\right)^{-1} d r^{2} \\
& +\frac{r^{4}}{b^{2}} d \Omega^{2}
\end{aligned}
$$

The spherical symmetry is restored in a SdS and a TdS black hole in the absence of a high energy mode in the quantum geometries. The emergent geometries in the low energy limit correspond to classical vacua presumably in Einstein gravity. The correct curvature in the classical regime is described by a Ricci scalar. It is worked out for a SdS black hole (86) and is given by

$R=\frac{20}{b^{2}}+\frac{6 N^{2}}{r^{4}}+\frac{2\left(b^{2}-r^{2}\right)}{b^{2} r^{4}}\left(b^{2}-7 r^{2}\right)$.

The curvature singularity at $r \rightarrow 0$ is forbidden by the black hole mass $N$ in the brane window. A priori the cosmological horizon $r_{c}=b$ appears to shrink and an event horizon $r_{e}=$ $N$ expands in a SdS black hole underlying a flow to set an equipotential sourced by a zero mode and a non-zero mode of a two form. It leads to a Nariai black hole and the observer lies along a time-like $r$. The limit $r_{e} \rightarrow r_{c}$ along a spatial $r$ or generically $r \rightarrow r_{c}$ may be analyzed for the Ricci scalar in a Nariai black hole to yield $R=\left(26 / b^{2}\right)$. Interestingly the near horizon geometry in a TdS black hole (87) may be approximated by $R=\left(20 / b^{2}\right)$. It is remarkable that a quantum TdS in the low energy limit reduces to a classical SdS black hole presumably underlying the Einstein vacuum. Similarly, a quantum SdS black hole in this limit reduces to a classical TdS black hole on a vacuum created pair of $(D \bar{D})_{3}$ branes. The aspects of quantum tunneling of a $\mathrm{SdS}$ vacuum to a TdS vacuum, via a Nariai black hole, argued in Ref. [16] may be invoked to resolve the apparent puzzle underlying a geometric transition between a quantum TdS (or SdS) and a classical SdS (or TdS).

\section{Concluding remarks}

An effective geometric torsion dynamics, underlying an irreducible curvature scalar in a second order formalism, is explored on a $D_{4}$-brane. In particular we have considered a two form in a $U(1)$ gauge theory on a $D_{4}$-brane in the presence of a background metric, which presumably describes an effective open string metric on an anti- $D_{4}$-brane. In other words the background metric is assumed to be sourced by the global modes of a NS two form in an open string world-sheet. It was argued that a local two form can generate a pair of $(D \bar{D})_{3}$-branes at the cosmological horizon of a background de Sitter vacuum with the Big Bang. The non-linear charge, underlying a dynamical two form, is believed to generate an effective geometry on a brane and an anti-brane in a pair. However, an observer in a brane universe is unaware of the existence of an anti-brane. Interestingly the effective curvature formalism on a $D_{4}$-brane turns out to be non-perturbative due to the $D$-instanton correction. Alternately a non-trivial geometry may be viewed by a pair of vacuum created $(D \bar{D})_{3}$ branes. Our analysis reveals an extra fifth dimension between 
a brane and an anti-brane world. The hidden dimension to a $D_{3}$-brane universe has been argued to play a significant role to explain the inflation observed in cosmology. Importantly the Poincare dual of the torsion, i.e. an axion, on an anti$D_{3}$-brane has been argued to describe quintessence in the formalism. Its dynamics on an anti- $D_{3}$-brane greatly influences the effective $D_{3}$-brane universe through the hidden fifth dimension. Quintessence is believed to be a potential candidate for the dark energy. It accounts for the time varying vacuum energy density observed by CMB in cosmology.

The formalism is explicitly worked out to describe various effective de Sitter quantum geometries underlying Schwarzschild, topological, and Reissner-Nordstrom black holes. The quantum black holes were shown to be free from a curvature singularity at $r \rightarrow 0$ due to the lower bound on the brane window. In the low energy limit the non-perturbative correction sourced by the torsion has been argued to be insignificant. The quantum black holes undergo expansion in the limit and reduce to the Einstein vacuum. In the context of a non-propagating two form on a $D_{2}$-brane it may be seen to possess its origin in a dynamical two form on a higher dimensional $D_{p}$-brane. Thus the existence of a BTZ black hole in three dimensions due to the non-zero (negative) vacuum energy density in Einstein gravity may be addressed by a dynamical two form. It may lead to an effective curvature on a $D_{p}$-brane for $p \geq 3$ underlying a ten dimensional type IIA or type IIB superstring theory on $S^{1}$. In principle the formalism may be generalized to investigate various versions of higher form dynamics on a space filling $D_{9}$-brane in superstring theory. It would be like a hint to the existence of an anti- $D_{9}$-brane. Arguably the global geometries on a vacuum created pair of $(D \bar{D})_{9}$-branes may provide a clue to the conjectured M-theory in 11 dimensions.

Acknowledgments The authors would like to thank Daya Shankar Kulshreshtha, Sudhakar Panda, Swarnendu Sarkar, and Ashoke Sen for various useful discussions. R.K. gratefully acknowledges the CSIR fellowship.

Open Access This article is distributed under the terms of the Creative Commons Attribution License which permits any use, distribution, and reproduction in any medium, provided the original author(s) and the source are credited.

Funded by $\mathrm{SCOAP}^{3}$ / License Version CC BY 4.0.
4. M. Mars, J.M.M. Senovilla, R. Vera, Phys. Rev. Lett. 86, 4219 (2001)

5. G.W. Gibbons, A. Ishibashi, Class. Quantum Gravity 21, 2919 (2004)

6. S. Kar, S. Majumdar, Phys. Rev. D 74, 066003 (2006)

7. S. Kar, S. Majumdar, Int. J. Mod. Phys. A 21, 6087 (2006)

8. S. Kar, S. Majumdar, Int. J. Mod. Phys. A 21, 2391 (2006)

9. S. Kar, Phys. Rev. D 74, 126002 (2006)

10. S. Kar, JHEP 0610, 052 (2006)

11. S. Kar, Int. J. Mod. Phys. A 24, 3571 (2009)

12. L.-H. Liu, B. Wang, G.-H. Yang, Phys. Rev. D 76, 064014 (2007)

13. J. Zhang, Phys. Lett. B 668, 353 (2008)

14. E.D. Grezia, G. Esposito, G. Miele, J. Phys. A 41, 164063 (2008)

15. E. Spallucci, A. Smailagic, P. Nicolini, Phys. Lett. B 670, 449 (2009)

16. A.K. Singh, K.P. Pandey, S. Singh, S. Kar, JHEP 05, 033 (2013)

17. A.K. Singh, K.P. Pandey, S. Singh, S. Kar, Phys. Rev. D 88, 066001 (2013)

18. A.K. Singh, K.P. Pandey, S. Singh, S. Kar, Nucl. Phys. B 251-252, $141(2014)$

19. K.P. Pandey, A.K. Singh, S. Singh, S. Kar, J. Astrophys. Aerosp. Technol. 03, 01 (2013)

20. S. Kar, J. Astrophys. Aerosp. Technol. 03, e106 (2013)

21. S. Singh, K.P. Pandey, A.K. Singh, S. Kar, Nucl. Phys. B 879, 216 (2014)

22. S. Singh, K.P. Pandey, A.K. Singh, S. Kar, Int. J. Mod. Phys. A (2014, in press)

23. S. Kar, K.P. Pandey, S. Singh, A.K. Singh, World Sci. Proc. C1002-24, 559 (2011)

24. A.K. Singh, K.P. Pandey, S. Singh, S. Kar, World Sci. Proc. C1002-24, 567 (2011)

25. S. Candelas, G.T. Horowitz, A. Strominger, Nucl. Phys. B 258, 46 (1985)

26. D.S. Freed, Commun. Math. Phys. 107, 483 (1986)

27. S.W. Hawking, Commun. Math. Phys. 43, 199 (1975)

28. S. Chen, J. Jing, Class. Quantum Gravity 22, 4651 (2005)

29. S.B. Chen, B. Wang, R.K. Su, Phys. Rev. D 77, 2401 (2008)

30. W. Yi-Huan, R. Jun, Chin. Phys. B 22, 030402 (2013)

31. R. Bousso, S. Hawking, Phys. Rev. D 57, 2436 (1998)

32. M. Banados, T. Brotz, M.E. Ortiz, Phys. Rev. D 59, 046002 (1999)

33. S. Hawking, J.M. Maldacena, A. Strominger, JHEP 05, 001 (2001)

34. R. Bousso, A. Maloney, A. Strominger, Phys. Rev. D 65, 104039 (2002)

35. R.-G. Cai, Nucl. Phys. B 628, 375 (2002)

36. A.J.M. Medved, Phys. Rev. D 66, 124009 (2002)

37. S. Kachru, R. Kallosh, A. Linde, S. Trivedi, Phys. Rev. D 68, 046005 (2003)

38. U.H. Danielsson, JHEP 0909, 114 (2009)

39. C. Damian, JHEP 1306, 109 (2013)

40. R. Baumann (2014), arXiv:1404.2601 [hep-th]

41. R. Blumenhagen (2014), arXiv:1404.3542 [hep-th]

42. F. Hassler, D. Lust, S. Massai (2014), arXiv:1405.2325 [hep-th]

43. D. Garfinkle, G.T. Horowitz, A. Strominger, Phys. Rev. D 43, 31403143 (1991)

\section{References}

1. J. Polchinski, Phys. Rev. Lett. 75, 4724 (1995)

2. N. Seiberg, E. Witten, JHEP 09, 032 (1999)

3. G.W. Gibbons, K. Hashimoto, JHEP 09, 013 (2000) 